\title{
Obtaining presentations from group actions without making choices
}

\author{
ANDREW PUTMAN
}

\begin{abstract}
Consider a group $G$ acting nicely on a simply-connected simplicial complex $X$. Numerous classical methods exist for using this group action to produce a presentation for $G$. For the case that $X / G$ is 2 -connected, we give a new method that has the novelty that one does not have to identify a fundamental domain for the action. Indeed, the resulting presentation is canonical in the sense that no arbitrary choices need to be made. It can be viewed as a nonabelian analogue of a simple result in the study of equivariant homology.
\end{abstract}

20F05, 57M07

\section{Introduction}

A classical theme in group theory is that if a group $G$ acts nicely on a simply-connected space $X$, then one can use that action to construct presentations for $G$. The investigation of presentations obtained in this way goes back to the $19^{\text {th }}$ century study of Fuchsian groups. One version of such a result (together with an extensive bibliography) can be found in the paper [7] of Brown, and this theory has since been subsumed into the study of Haefliger's theory of complexes of groups [6].

All these classical results require the identification of a fundamental domain for the action. While some choice of this type is usually necessary for the resulting presentation to be finite, there often isn't a canonical choice of fundamental domain (this is similar to the fact that most vector spaces do not have a canonical basis). Moreover, especially if the action is not cocompact, it can be very difficult to identify a fundamental domain in a manner explicit enough for the machinery to work. In this paper, we show how to construct a presentation from a group action without identifying a fundamental domain, and more generally without making any arbitrary choices.

Statement of theorem Let $G$ be a group and $X$ be a simply connected simplicial complex upon which $G$ acts. We will assume that $G$ acts without rotations, that is, for all simplices $s$ of $G$ the stabilizer $G_{s}$ stabilizes $s$ pointwise (this can always be arranged to hold by subdividing $X$ ). An elementary argument of Armstrong [1] 
(recalled below in Section 3.1) shows that if $X / G$ is simply connected, then $G$ is generated by elements which stabilize vertices. In other words, we have a surjective map

$$
\underset{v \in X^{(0)}}{*} G_{v} \longrightarrow G
$$

As notation, if $g \in G$ stabilizes $v \in X^{(0)}$, then we denote $g$ considered as an element of

$$
G_{v}<\underset{v \in X^{(0)}}{*} G_{v}
$$

by $g_{v}$. There are then some obvious elements in the kernel of this map, which we write as relations $f=g$ rather than as elements $f g^{-1}$. First, we have $g_{v}=g_{v^{\prime}}$ if $v$ and $v^{\prime}$ are joined by an edge $e$ and $g \in G_{e}$. We call these the edge relations. Second, we have $g_{v} h_{w} g_{v}^{-1}=\left(g h g^{-1}\right)_{g(w)}$ for $g \in G_{v}$ and $h \in G_{w}$. We call these relations the conjugation relations.

The following theorem says that if $X / G$ is 2 -connected, then these two families of relations suffice to give a presentation.

Theorem 1.1 (Main theorem) Let a group $G$ act without rotations on a simply connected simplicial complex $X$. Assume that $X / G$ is 2-connected. Then

$$
G=\left(\underset{v \in X^{(0)}}{*} G_{v}\right) / R
$$

where $R$ is the normal subgroup generated by the conjugation relations and the edge relations.

Remark If $X / G$ is 1 -connected but not 2-connected, then one would also need relations corresponding to generators for $\pi_{2}(X / G)$. One could extract the precise form of such relations from our proof of Theorem 1.1. We leave this as an exercise for the interested reader.

Remark The conclusion of Theorem 1.1 resembles what occurs for groups acting with strict fundamental domains (see Bridson and Haefliger [6]). Consider a group $G$ acting without rotations on a 1 -connected simplicial complex $X$. A subcomplex $C \subset X$ is a strict fundamental domain if it contains exactly one point from each $G$-orbit. We then necessarily have $C \cong X / G$. The theory of complexes of groups shows that in this situation, the group $G$ can be constructed as a pushout of the stabilizers of simplices in $C$. However, Theorem 1.1 requires all of the stabilizer subgroups, and indeed one cannot merely use the stabilizers of vertices representing the orbits of $G$ (in particular, the assumptions of Theorem 1.1 do not imply that $G$ has a strict fundamental domain). 
This subtlety already shows up in Armstrong's theorem mentioned above: one really needs all the vertex stabilizers to generate $G$. See the remark after the second example below.

Examples We now give four examples of group actions to which Theorem 1.1 can be applied. The first two are easy toy examples and the last two are more substantial.

Example (Amalgamated free products) If $G=A *_{C} B$, then Bass-Serre theory [20] shows that there is a tree $X$ (that is, a simply connected 1-dimensional simplicial complex) upon which $G$ acts without rotations. The tree $X$ has the following two properties.

- $X / G$ is an edge $e$.

- There exists a lift $\tilde{e}$ of $e$ to $X$ such that if $v, w \in X^{(0)}$ are the vertices of $\tilde{e}$, then

$$
G_{v}=A, \quad G_{w}=B \quad \text { and } \quad G_{\tilde{e}}=C .
$$

The conditions of Theorem 1.1 are clearly satisfied. In the resulting presentation, the stabilizers of vertices correspond to the conjugates of $A$ and $B$ inside $G$.

Example (Symmetric group) Consider the symmetric group $S_{n}$ on the set $\{1, \ldots, n\}$ of $n$ letters. For distinct $a, b \in\{1, \ldots, n\}$, denote by $\tau_{a, b}$ the transposition of $a$ and $b$. We will briefly describe how to use Theorem 1.1 to prove that $S_{n}$ has the following presentation.

- The generating set $S$ is $\left\{\tau_{a, b} \mid a, b \in\{1, \ldots, n\}\right.$ distinct $\}$.

- The relations consist of the following for all distinct $a, b \in\{1, \ldots, n\}$ and $s \in S$ :

$$
\tau_{a, b}=\tau_{b, a}, \quad \tau_{a, b}^{2}=1 \quad \text { and } \quad s \tau_{a, b} s^{-1}=\tau_{s(a), s(b)}
$$

The proof is by induction on $n$. The base cases are $n \leq 3$, where the presentation is easily verified. Assume now that $n>3$.

Define $P_{n}$ to be the following poset. The elements of $P_{n}$ are ordered sequences $\sigma=\left(x_{1}, \ldots, x_{m}\right)$, where the $x_{i}$ are distinct elements of $\{1, \ldots, n\}$. For $\sigma, \sigma^{\prime} \in P_{n}$, we have $\sigma \leq \sigma^{\prime}$ if $\sigma$ is a subsequence of $\sigma^{\prime}$. Next, let $X_{n}$ be the geometric realization of $P_{n}$, that is, the simplicial complex whose $k$-simplices are totally ordered chains

$$
\sigma_{0}<\sigma_{1}<\cdots<\sigma_{k}
$$

of elements of $P_{n}$. It is clear that $S_{n}$ acts on $X_{n}$ without rotations. Björner and Wachs [5] proved that $X_{n}$ is $(n-2)$-connected. It is not hard to show that $X_{n} / S_{n}$ is 
also $(n-2)$-connected (we omit the proof since it is tangential to the remainder of the paper). We can thus apply Theorem 1.1 to the action of $S_{n}$ on $X_{n}$.

If $\sigma=\left(x_{1}, \ldots, x_{m}\right) \in P_{n}$, then $\left(S_{n}\right)_{\sigma}$ is required to fix $\left\{x_{1}, \ldots, x_{m}\right\}$ pointwise. It is thus the permutation group of $\{1, \ldots, n\} \backslash\left\{x_{1}, \ldots, x_{m}\right\}$, a set with $n-m \leq n-1$ elements. By induction, these stabilizer subgroups have presentations of the desired form. The edge relations identify identical transpositions that lie in different stabilizer subgroups, and after performing these identifications the conjugation relations become the relations $s \tau_{a, b} s^{-1}=\tau_{s(a), s(b)}$ from our presentation. We conclude that $S_{n}$ has a presentation of the desired form.

Remark For $k \geq 1$, let $P_{n}^{k}$ be the subposet of $P_{n}$ consisting of ordered sequences $\left(x_{1}, \ldots, x_{m}\right)$ with $m \geq k$. Also, let $X_{n}^{k}$ be the geometric realization of $P_{n}^{k}$. The paper of Björner and Wachs mentioned in the previous paragraph proves that $X_{n}^{k}$ is $(n-k-1)-$ connected, and again it is not hard to show that $X_{n}^{k} / S_{n}$ is $(n-k-1)$-connected as well. Setting $k=n-2$, the spaces $X_{n}^{k}$ and $X_{n}^{k} / S_{n}$ are both 1 -connected, so we can apply Armstrong's theorem (mentioned before the statement of Theorem 1.1) to get that $S_{n}$ is generated by stabilizers of $\sigma \in P_{n}^{k}$. It is clear that stabilizers of length $n-1$ and length $n$ sequences are trivial. If $\sigma=\left(x_{1}, \ldots, x_{k}\right) \in P_{n}^{k}$, then the stabilizer $\left(S_{n}\right)_{\sigma}$ is the permutation group of $\{1, \ldots, n\} \backslash \sigma$, a set with 2 elements. We therefore recover (in a maximally complicated manner) the fact that $S_{n}$ is generated by transpositions. Since $S_{n}$ acts transitively on elements $\left(x_{1}, \ldots, x_{k}\right)$ of $P_{n}^{k}$ but is not generated by fewer than $n-1$ transpositions, this illustrates the fact that we really need all the vertex stabilizers and not just representatives of each vertex orbit.

Example (Torelli group) In [19], the author uses Theorem 1.1 to obtain a natural infinite presentation of the Torelli subgroup of the mapping class group of a surface. Recall that the mapping class group $\operatorname{Mod}_{g}$ is the group of homotopy classes of orientationpreserving diffeomorphisms of a closed orientable genus $g$ surface $\Sigma_{g}$. The Torelli group $\mathcal{I}_{g}<\operatorname{Mod}_{g}$ is the kernel of the action of $\operatorname{Mod}_{g}$ on $\mathrm{H}_{1}\left(\Sigma_{g} ; \mathbb{Z}\right)$. The complex used in [19] is a variant on the complex of curves, which is the simplicial complex whose simplices are sets $\left\{\gamma_{0}, \ldots, \gamma_{k}\right\}$ of nontrivial homotopy classes of simple closed curves on $\Sigma_{g}$ that can be realized disjointly.

Remark While it is known from work of McCool [14] (see also the paper by Hatcher and Thurston [10]) that $\operatorname{Mod}_{g}$ is finitely presentable for all $g$, it is not known whether or not $\mathcal{I}_{g}$ is finitely presentable for $g \geq 3$. All that is known is that it is finitely generated for $g \geq 3$ (see Johnson [11]) and is not finitely generated for $g=2$ (see McCullough and Miller [15] and Mess [16]). 
Example (Congruence subgroups) In [18], the author uses Theorem 1.1 to obtain natural finite presentations for the level 2 and 3 principal congruence subgroups of $\mathrm{SL}_{n}(\mathbb{Z})$. Recall that the level $L$ principal congruence subgroup $\Gamma_{n}(L)$ of $\mathrm{SL}_{n}(\mathbb{Z})$ is the kernel of the natural map $\mathrm{SL}_{n}(\mathbb{Z}) \rightarrow \mathrm{SL}_{n}(\mathbb{Z} / L)$. Finite presentations for $\mathrm{SL}_{n}(\mathbb{Z})$ were first found by Magnus (see Milnor [17]), but the presentations in [18] seem to be the first presentations for $\Gamma_{n}(L)$ in the literature. The complex used in [18] is as follows. Let $\mathcal{B}_{n}$ be the simplicial complex whose simplices are sets $\left\{v_{0}, \ldots, v_{k}\right\}$ of vectors in $\mathbb{Z}^{n}$ which form part of a basis for $\mathbb{Z}^{n}$. Work of Maazen [13] shows that $\mathcal{B}_{n}$ and $\mathcal{B}_{n} / \Gamma_{n}(L)$ are $(n-2)$-connected for $L \geq 2$. This shows that $\mathcal{B}_{n}$ satisfies the conditions of Theorem 1.1 for $n \geq 4$. The inductive argument starts with the base case $n=2$; for $n=3$, additional 3 -cells are attached to $\mathcal{B}_{n}$ to make the quotient $2-$ connected.

Remark Of course, Theorem 1.1 only yields an infinite presentation for $\Gamma_{n}(L)$. Additional techniques are needed to reduce this to a simple finite presentation (this is where the restriction $L \in\{2,3\}$ is used).

Comments on proof Though there are numerous methods for obtaining group presentations from group actions, we have been unable to derive Theorem 1.1 from any of the ones of which we are aware. Rather, our starting point is the theorem of Armstrong discussed above, which we recall uses the assumption that $X / G$ is simply-connected to show that the natural map

$$
\psi:\left(\underset{v \in X^{(0)}}{*} G_{v}\right) / R \longrightarrow G
$$

is surjective. In fact, Armstrong gives an algorithm (which we call the "Armstrong construction") which takes an element of $G$ and expresses it in terms of vertex stabilizers. Numerous choices need to be made when running this algorithm. However, via a careful analysis of the combinatorics of homotoping loops and discs inside simplicial complexes we will show that modulo our relations none of these choices matter. The upshot is that the Armstrong construction induces a well defined surjective map

$$
\psi^{-1}: G \longrightarrow\left(\underset{v \in X^{(0)}}{*} G_{v}\right) / R
$$

satisfying $\psi \circ \psi^{-1}=1$.

Remark Our proof is inspired in part by another paper of Armstrong [2] in which he gives a combinatorial-topological derivation of the presentation of Brown [7] alluded to in the first paragraph of this paper. Brown originally derived his presentation via Bass-Serre theory. 
Motivation from equivariant topology Theorem 1.1 can be viewed as a nonabelian analogue of a simple result in equivariant topology. We proceed to sketch this. Nothing in this section is needed in the rest of the paper.

Let $G$ and $X$ be as in Theorem 1.1. We will assume that $X / G$ is the simplicial complex whose simplices are orbits of simplices of $X$ (this can always be arranged by subdividing $X$; we remark that it does not follow from the fact that $G$ acts without rotations). Let $Y$ be the 1 -skeleton of $X / G$. Regarding the simplicial complex $Y$ as a category whose objects are simplices and whose morphisms are inclusions, there is a natural contravariant functor $\mathcal{H}_{1}$ from $Y$ to the category of abelian groups. Namely, if $s$ is a simplex of $Y$, then $\mathcal{H}_{1}(s)=\mathrm{H}_{1}\left(G_{\widetilde{s}} ; \mathbb{Z}\right)$, where $\tilde{s}$ is a lift of $s$ to $X$. Define $\mathcal{C}\left(\mathcal{H}_{1}\right)$ to be the colimit of $\mathcal{H}_{1}$; that is, the quotient of the abelian group

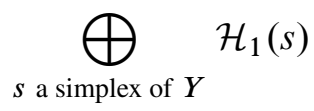

which for all edges $e=\left\{v, v^{\prime}\right\}$ of $Y$ identifies $\mathcal{H}_{1}(e)$ with its images in $\mathcal{H}_{1}(v)$ and $\mathcal{H}_{1}\left(v^{\prime}\right)$.

Let $\mathrm{H}_{k}^{G}(X ; \mathbb{Z})$ denote the equivariant homology groups of $G$ acting on $X$ in the sense of Brown [8, §VII.7] (defined in terms of the Borel construction). These satisfy the following two properties.

- Since $\pi_{1}(X)=1$, the spectral sequence (7.2) of [8, §VII.7] implies that $\mathrm{H}_{1}^{G}(X ; \mathbb{Z}) \cong \mathrm{H}_{1}(G ; \mathbb{Z})$.

- The spectral sequence whose $E^{2}$ page is described in $[8, \S$ VII.8] is a firstquadrant spectral sequence, so it induces a 5-term exact sequence. This exact sequence takes the form

(1) $\mathrm{H}_{2}^{G}(X ; \mathbb{Z}) \longrightarrow \mathrm{H}_{2}(X / G ; \mathbb{Z}) \longrightarrow \mathcal{C}\left(\mathcal{H}_{1}\right) \longrightarrow \mathrm{H}_{1}^{G}(X ; \mathbb{Z}) \longrightarrow \mathrm{H}_{1}(X / G ; \mathbb{Z}) \longrightarrow 0$.

Since $X / G$ is $2-$ connected, (1) implies that

$$
\mathrm{H}_{1}(G ; \mathbb{Z}) \cong \mathrm{H}_{1}^{G}(X ; \mathbb{Z}) \cong \mathcal{C}\left(\mathcal{H}_{1}\right) .
$$

One can view the group presentation in Theorem 1.1 as a nonabelian analogue of $\mathcal{C}\left(\mathcal{H}_{1}\right)$, and in fact (2) can be easily deduced from Theorem 1.1.

Remark In fact, our original motivation for guessing that something like Theorem 1.1 might be true was van den Berg's unpublished thesis [3], where she used (2) to give a new proof of Johnson's theorem [12] giving the abelianization of the Torelli subgroup of the mapping class group. 
Acknowledgments This paper is a revised version of part of my thesis, and I wish to thank my advisor Benson Farb for his help and encouragement. Additionally, I wish to thank Anne Thomas, Shmuel Weinberger, and Ben Wieland for helpful conversations.

\section{Simplicial Preliminaries}

The heart of our proof is a careful examination of the combinatorics of homotoping loops and discs in simplicial complexes. In this section, we establish some preliminary results in this direction.

\subsection{Simplicial complexes}

We first establish our notation for simplicial complexes. Let $X$ be a simplicial complex. We will denote by $|X|$ the geometric realization of $X$. When we say that a set $\sigma=\left\{x_{0}, \ldots, x_{k}\right\}$ of vertices of $X$ forms a $k$-simplex, we allow the possibility that $x_{i}=x_{j}$ for some $i$ and $j$. By a path or a loop in $X$, we mean a simplicial path or loop in the 1 -skeleton. We will denote the path or loop that starts at a vertex $v_{0}$, goes along an edge to a vertex $v_{1}$, then goes along an edge to a vertex $v_{2}$, etc. and ends at a vertex $v_{n}$ (which equals $v_{0}$ if the path is a loop) by $v_{0}-v_{1}-\cdots-v_{n}$. To simplify our notation, we will allow the possibility that $v_{i}=v_{i+1}$ for some $0 \leq i<n$ (this is consistent with regarding $\{v, v\}$ as a degenerate edge for a vertex $v$ of $X$ ). However, we will not regard such a path as injective (for example, in the definition of degenerate discs below in Section 2.2). If $f: X \rightarrow Y$ is a map of simplicial complexes and $\gamma$ is a path or loop in $X$, then we will denote by $f_{*}(\gamma)$ the induced path or loop in $Y$. Finally, if $s$ is a simplex of $X$, then the star of $s$, denoted $\operatorname{Star}_{X}(s)$, is the subcomplex of $X$ consisting of all simplices $s^{\prime}$ such that there is a simplex $s^{\prime \prime}$ of $X$ containing both $s$ and $s^{\prime}$ as faces.

\subsection{Collapsing loops via degenerate discs}

While simplicially contracting paths to points, we will need the notion of a degenerate disc. To define this, we need a pair of preliminary definitions.

Definition 2.1 A space/loop pair is a pair $(X, \gamma)$, where $X$ is a simplicial complex with a basepoint $*$ and $\gamma$ is a loop in $X$ that is based at $*$.

Definition 2.2 A nondegenerate disc is a space/loop pair $(D, \gamma)$ with the following properties.

- $\quad D$ is homeomorphic to a closed 2-disc. 
- The basepoint $* \in D$ lies in $\partial D$.

- $\gamma$ is an injective loop in $\partial D$ that is based at $*$ and goes once around $\partial D$.

Definition 2.3 A degenerate disc (see Figure 1(b)) is a space/loop pair $(D, \gamma)$ such that there exists a sequence $\left(D_{1}, \gamma_{1}\right), \ldots,\left(D_{k}, \gamma_{k}\right)$ of space/loop pairs with the following properties.

- $\left(D_{1}, \gamma_{1}\right)$ is a nondegenerate disc and $\left(D_{k}, \gamma_{k}\right)=(D, \gamma)$.

- For $1 \leq i<k$, the space/loop pair $\left(D_{i+1}, \gamma_{i+1}\right)$ is obtained from $\left(D_{i}, \gamma_{i}\right)$ by one of the following two moves. Let $*$ be the basepoint of $D_{i}$.

- If $\gamma_{i}$ contains a subpath of the form $x_{1}-x_{2}$ for vertices $x_{1}, x_{2} \in D_{i}^{(0)}$ and there is some vertex $y \in D_{i}^{(0)}$ such that $\left\{x_{1}, x_{2}, y\right\}$ is a 2-simplex of $D_{i}$ and $y \neq x_{1}, x_{2}$ (see Figure 1(a)), then we can homotope $x_{1}-x_{2}$ to $x_{1}-y-x_{2}$ and delete $\left\{x_{1}, x_{2}\right\}$ and $\left\{x_{1}, x_{2}, y\right\}$ from $D_{i}$. We will call this a two-dimensional collapse.

- If $\gamma_{i}$ contains a subpath of the form $x_{1}-x_{2}-x_{1}$ for vertices $x_{1}, x_{2} \in D_{i}^{(0)}$ with $x_{2} \neq x_{1}$, * (see Figure 1(a)), then we can homotope $x_{1}-x_{2}-x_{1}$ to the constant path $x_{1}$ and delete $\left\{x_{1}, x_{2}\right\}$ from $D_{i}$. We will call this a one-dimensional collapse.

We will use the term collapse to refer to either a one- or two-dimensional collapse.

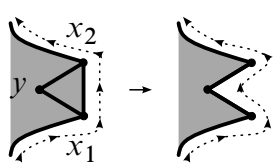

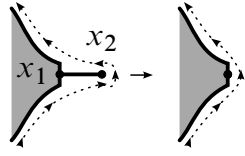

(a)

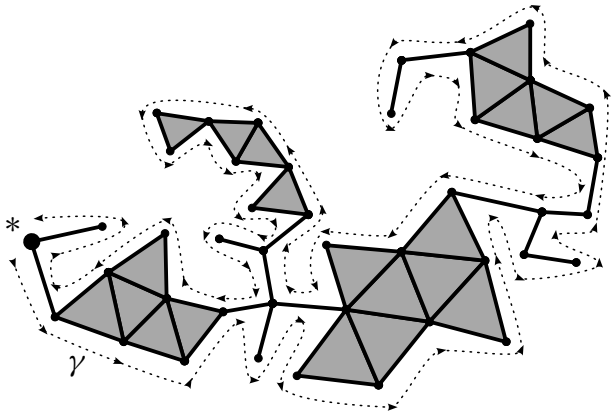

(b)

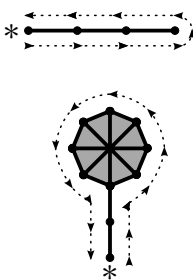

(c)

Figure 1: (a) Two- and one-dimensional collapses (b) A degenerate disc

(c) A line and a flower

Remark It is easy to see that the one- and two-dimensional collapses involved in the definition of a degenerate disc do not delete simplices that appear elsewhere in the loop in question. 
Remark The intermediate space/loop pairs $\left(D_{i}, \gamma_{i}\right)$ in the definition of a degenerate discs are themselves degenerate discs, but need not be nondegenerate discs.

Remark The cell complexes used in van Kampen diagrams are (almost) degenerate discs, the only difference being that we require each cell to be a triangle.

A key technical lemma concerning degenerate discs is as follows.

Lemma 2.4 Let $(D, \gamma)$ be a degenerate disc and let $X$ be a subcomplex of $D$ which is homeomorphic to a closed 2-disc. Let $e$ be an edge of $\partial X$ that does not lie in $\gamma$. There then exists a sequence $s_{1}, \ldots, s_{k}$ of 2 -simplices of $D$ with the following properties.

- $s_{i}$ is not a 2-simplex of $X$ for $1 \leq i \leq k$.

- $s_{i} \cap s_{i+1}$ is an edge of $D$ for $1 \leq i<k$.

- $\quad e$ is an edge of $s_{1}$ and $s_{k}$ contains an edge of $\gamma$.

Proof Assume first that $(D, \gamma)$ is a nondegenerate disc. Observe that each component of $|D| \backslash|X|$ must contain an edge of $\gamma$. We can therefore choose a continuous path $\delta$ in $|D| \backslash$ Interior $(|X|)$ which begins at an interior point of $e$ and ends at an interior point of an edge of $\gamma$. Moreover, we can choose $\delta$ such that it does not pass through any vertices of $D$, such that all of its intersections with edges of $D$ are transverse, and such that it only intersects finitely many 2 -simplices of $D$. The desired sequence of 2 -simplices is then the sequence of 2 -simplices through which $\delta$ passes.

We now consider the general case. It is enough to show that if the lemma is true for some degenerate disc $\left(D^{\prime}, \gamma^{\prime}\right)$, then it remains true for the result $(D, \gamma)$ of performing a collapse to $\left(D^{\prime}, \gamma^{\prime}\right)$. The case of a one-dimensional collapse being trivial, we consider the case that $(D, \gamma)$ is the result of performing a two-dimensional collapse to $\left(D^{\prime}, \gamma^{\prime}\right)$. Let $t$ be the 2-simplex of $D^{\prime}$ that is collapsed. Consider a subcomplex $X$ and an edge $e$ of $D$ as in the lemma. Regarding $D$ and thus $X$ as a subcomplex of $D^{\prime}$, by assumption there is a sequence $s_{1}, \ldots, s_{k}$ of 2-simplices of $D^{\prime}$ satisfying the conditions of the lemma (applied to the subcomplex $X$ in the degenerate disc $\left(D^{\prime}, \gamma^{\prime}\right)$ ). Observe that since $e$ is not an edge of $\gamma$, it follows that $s_{1} \neq t$. Set

$$
l=\max \left\{i \mid 1 \leq i \leq k, s_{j} \neq t \text { for } 1 \leq j \leq i\right\} .
$$

It is then clear that the sequence $s_{1}, \ldots, s_{l}$ of 2 -simplices of $D$ satisfies the conditions of the lemma, and we are done. 
Using Lemma 2.4, we can show that degenerate discs can always be contracted to points by a sequence of collapses. This is a standard result, but we give the short proof to make this paper self-contained. The reader should be warned that analogous statements are false in dimensions greater than 2 (see Bing [4, §4, Example 3] and Goodrick [9]).

Corollary 2.5 Let $(D, \gamma)$ be a degenerate disc. Then we can perform a sequence of collapses to $(D, \gamma)$ such that the resulting degenerate disc $\left(D^{\prime}, \gamma^{\prime}\right)$ consists of a single vertex $*$ together with the constant path $\gamma^{\prime}=*$.

Proof If $D$ contains any 2-simplex, then by Lemma 2.4 there is some $2-$ simplex containing an edge of $\gamma$. We can therefore perform a sequence of two-dimensional collapses so as to obtain a degenerate disc $\left(D^{\prime}, \gamma^{\prime}\right)$ such that $D^{\prime}$ is a simply-connected 1-complex; that is, a tree. We can then perform a sequence of one-dimensional collapses to collapse $D^{\prime}$ to $*$, and we are done.

Finally, the following two special types of degenerate discs will play a key role in our proofs.

Definition 2.6 A line (see top of Figure 1(d)) is a degenerate disc $(D, \gamma)$ of the following form.

- $\quad D$ is the complex consisting of vertices $v_{1}, \ldots, v_{n}$ for some $n \geq 1$ together with edges between $v_{i}$ and $v_{i+1}$ for $1 \leq i<n$. The basepoint $*$ is $v_{1}$. If $n \geq 2$, then the edge $v_{n-1}-v_{n}$ will be called the final edge.

- $\gamma$ is the path $v_{1}-\cdots-v_{n-1}-v_{n}-v_{n-1}-\cdots-v_{1}$.

A flower (see bottom of Figure 1(d)) is a degenerate disc $(D, \gamma)$ of the following form.

- There is a nondegenerate disc $\left(D^{\prime}, \gamma^{\prime}\right)$ satisfying the following condition. The complex $D^{\prime}$ is a subcomplex of $D$ and for some $n \geq 1$ the complex $D^{\prime}$ consists of $D$, vertices $v_{1}, \ldots, v_{n-1}$, and edges between $v_{i}$ and $v_{i+1}$ for $1 \leq i<n$, where $v_{n}$ is the basepoint of $\left(D^{\prime}, \gamma^{\prime}\right)$. The basepoint of $(D, \gamma)$ is $v_{1}$.

- $\gamma^{\prime}$ is the path $v_{1}-\cdots-v_{n}-\gamma-v_{n}-\cdots-v_{1}$.

We will call $\left(D^{\prime}, \gamma^{\prime}\right)$ the bloom of $(D, \gamma)$ and the path $v_{1}-\cdots-v_{n}$ the stem; we will confuse the stem with its associated subcomplex.

Remark A line may consist of a single vertex. Similarly, the stem of a flower may consist of a single vertex, in which case the bloom is the entire flower. 
The following corollary to Lemma 2.4 will be frequently used, and is the key reason we introduced lines and flowers.

Corollary 2.7 Let $(D, \gamma)$ be a degenerate disc.

(1) Let $e$ be an edge of $D$. Let $\left(D^{\prime}, \gamma^{\prime}\right)$ be a minimal degenerate disc with $e$ an edge of $D^{\prime}$ to which we can collapse $(D, \gamma)$. Then $\left(D^{\prime}, \gamma^{\prime}\right)$ is a line whose final edge is $e$.

(2) Let $X$ be a subcomplex of $D$ which is homeomorphic to a closed 2-disc. Let $\left(D^{\prime}, \gamma^{\prime}\right)$ be a minimal degenerate disc with $X$ a subcomplex of $D^{\prime}$ to which we can collapse $(D, \gamma)$. Then $\left(D^{\prime}, \gamma^{\prime}\right)$ is a flower whose bloom consists of $\left(X, \gamma^{\prime \prime}\right)$ for some path $\gamma^{\prime \prime}$ around the boundary of $X$. If $X$ contains the basepoint $*$ of $(D, \gamma)$, then in fact $D^{\prime}=X$.

Proof The proofs of the two conclusions are similar; we will prove the more difficult second one and leave the first to the reader.

The first step is to prove that any 2-simplex of $D^{\prime}$ is contained in $X$. Assume otherwise. If $\partial X$ contains an edge $e$ which is not contained in $\gamma^{\prime}$, then by Lemma 2.4 (applied to $X$ and $e$ ), there must exist some 2-simplex $s$ such that $s$ is not a 2-simplex of $X$ and such that $s$ contains an edge of $\gamma^{\prime}$. We can then perform a two-dimensional collapse so as to eliminate $s$, contradicting the minimality of $\left(D^{\prime}, \gamma^{\prime}\right)$. All the edges of $\partial X$ must therefore be contained in $\gamma^{\prime}$.

An easy induction establishes that if $(E, \delta)$ is any degenerate disc and $\varepsilon$ is an edge of $\delta$, then at most one 2-simplex of $E$ contains $\varepsilon$. We conclude that there does not exist a $2-$ simplex of $D^{\prime}$ whose intersection with $X$ is an edge. Applying Lemma 2.4 again, we conclude that since there exists a 2-simplex $t$ of $D^{\prime}$ that does not lie in $X$, there must exist a 2-simplex $t^{\prime}$ of $D^{\prime}$ that does not lie in $X$ such that $t^{\prime}$ contains an edge of $\gamma^{\prime}$. We can therefore perform a two-dimensional collapse so as to eliminate $t^{\prime}$, again contradicting the minimality of $\left(D^{\prime}, \gamma^{\prime}\right)$. We conclude that every 2-simplex of $D^{\prime}$ is in fact contained in $X$.

Since $D^{\prime}$ and $X$ are contractible, the space $D^{\prime} / X$ is contractible. Since $D^{\prime} / X$ contains no 2-cells, it follows that $D^{\prime} / X$ is a tree. We conclude that the closure of every component of $\left|D^{\prime}\right| \backslash|X|$ must be a tree that intersects $X$ in exactly one point. Since trees can be collapsed to any of their vertices, we deduce that $\left|D^{\prime}\right| \backslash|X|$ can have at most one component (the one containing the basepoint of $\left(D^{\prime}, \gamma^{\prime}\right)$; this exists exactly when $X$ does not contain the basepoint of $\left.\left(D^{\prime}, \gamma^{\prime}\right)\right)$. Moreover, if there is such a component, then its closure must satisfy the conditions of the stem of a flower, and we are done. 


\subsection{Simplicial homotopy}

We now give moves by which we will homotope discs in simplicial complexes. The following definition is a variant on the notion of contiguity classes in Spanier [21].

Definition 2.8 Let $(K, L)$ be a simplicial pair, let $X$ be a simplicial complex, and let $f: K \rightarrow X$ be a simplicial map.

- Let $s$ be an $n$-simplex of $K$ with $n \geq 1$ and let $v$ be a vertex of $s$. Let $\left(K^{\prime}, L^{\prime}\right)$ be the result of subdividing the simplicial pair $(K, L)$ by adding a vertex $v^{\prime}$ to the interior of $s$, and define $f^{\prime}: K^{\prime} \rightarrow X$ by setting

$$
f^{\prime}(w)=\left\{\begin{array}{ll}
f(w) & \text { if } w \neq v^{\prime} \\
f(v) & \text { if } w=v^{\prime}
\end{array} \quad\left(w \in\left(K^{\prime}\right)^{(0)}\right)\right.
$$

and extending linearly. We will say that the complex $\left(K^{\prime}, L^{\prime}\right)$ and the map $f^{\prime}: K^{\prime} \rightarrow X$ come from performing an elementary subdivision of $f$ along $s$ with $v$.

- Let $v \in K^{(0)} \backslash L^{(0)}$ and $x \in X^{(0)}$ be such that for all simplices $s$ of $\operatorname{Star}_{K}(v)$, the set $f(s) \cup\{x\}$ is a simplex of $X$. Define $f^{\prime}: K \rightarrow X$ by setting

$$
f^{\prime}(w)=\left\{\begin{array}{ll}
f(w) & \text { if } w \neq v \\
x & \text { if } w=v
\end{array} \quad\left(w \in K^{(0)}\right)\right.
$$

and extending linearly. We will say that the map $f^{\prime}: K \rightarrow X$ comes from performing an elementary push of $f$ along $v$ with $x$.

- If $f^{\prime}: K \rightarrow X$ differs from $f$ by a sequence of elementary pushes, then we say that $f$ and $f^{\prime}$ are elementarily equivalent.

We will need the following theorem. It is essentially [21, Theorem 3.5.6], but since our definitions are a little different we include a sketch of the proof.

Theorem 2.9 (Spanier [21, Theorem 3.5.6]) Let $(K, L)$ be a compact simplicial pair, let $X$ be a simplicial complex, and let $f_{0}, f_{1}: K \rightarrow X$ be simplicial maps satisfying the following conditions.

- $\left.f_{0}\right|_{L}=\left.f_{1}\right|_{L}$.

- The maps $f_{0}$ and $f_{1}$ are homotopic fixing $L$; that is, there is a continuous map $F:|K| \times[0,1] \rightarrow|X|$ such that $F(\cdot, 0)=f_{0}$, such that $F(\cdot, 1)=f_{1}$, and such that $F(x, t)=f_{0}(x)$ for $x \in|L|$. 
Then there exists a subdivision $\left(K^{\prime}, L^{\prime}\right)$ of $(K, L)$ and simplicial maps $f_{1}^{\prime}, f_{2}^{\prime}: K \rightarrow X$ which are obtained from the $f_{i}$ by sequences of elementary subdivisions such that $f_{1}^{\prime}$ and $f_{2}^{\prime}$ are elementarily equivalent.

Proof Since $K$ is compact, a Lebesgue number argument implies that there exists a sequence $0=t_{0}<t_{1}<\cdots<t_{n}=1$ such that for $x \in|K|$ and $1 \leq i \leq n$, there exists a vertex $v \in X^{(0)}$ such that $F\left(x, t_{i-1}\right), F\left(x, t_{i}\right) \in \operatorname{Interior}\left(\operatorname{Star}_{X}(v)\right)$. For $0 \leq i \leq n$, define $g_{i}:|K| \rightarrow|X|$ by $g_{i}(x)=F\left(x, t_{i}\right)$ for $x \in|K|$, so $g_{0}=f_{0}$ and $g_{n}=f_{1}$.

By construction, for $1 \leq i \leq n$ the set

$$
\mathcal{U}_{i}:=\left\{g_{i-1}^{-1}\left(\operatorname{Interior}\left(\operatorname{Star}_{X}(v)\right)\right) \cap g_{i}^{-1}\left(\operatorname{Interior}\left(\operatorname{Star}_{X}(v)\right)\right) \mid v \in X^{(0)}\right\}
$$

is an open cover of $K$. Let $\left(K^{\prime}, L^{\prime}\right)$ be a subdivision of $(K, L)$ which is finer than $\mathcal{U}_{i}$ for $1 \leq i \leq n$. For $0 \leq i \leq n$, we can construct simplicial maps $\phi_{i}: K^{\prime} \rightarrow X$ satisfying the following conditions.

(1) $\phi_{0}$ and $\phi_{n}$ can be obtained by a sequence of elementary subdivisions from $f_{0}$ and $f_{1}$, respectively.

(2) For all vertices $v \in\left(K^{\prime}\right)^{(0)}$, we have

$$
f_{i-1}\left(\operatorname{Star}_{K^{\prime}}(v)\right) \cup f_{i}\left(\operatorname{Star}_{K^{\prime}}(v)\right) \subset \operatorname{Star}_{X}\left(\phi_{i}(v)\right)
$$

for $1 \leq i \leq n$.

(3) For all vertices $v \in\left(L^{\prime}\right)^{(0)}$, we have $\phi_{i}(v)=\phi_{j}(v)$ for all $0 \leq i, j \leq n$.

For $1 \leq i \leq n$, items 2 and 3 imply that $\phi_{i}$ is elementarily equivalent to $\phi_{i-1}$. We conclude that $\phi_{0}$ is elementarily equivalent to $\phi_{n}$, so $f_{1}^{\prime}=\phi_{0}$ and $f_{2}^{\prime}=\phi_{n}$ satisfy the conditions of the theorem.

\section{Proof of the main theorem}

We begin by observing that since $G$ acts on $X$ without rotations, we can subdivide $X$ without affecting the conclusion of the theorem. Indeed, subdividing introduces new vertices, but the stabilizer $G_{x^{\prime}}$ of a new vertex $x^{\prime}$ lies in $G_{x}$ for some old vertex $x$, and the edge relations identify $G_{X^{\prime}}$ with its image in $G_{x}$. Moreover, it is easy to see that all the new edge and conjugation relations involving elements of $G_{X^{\prime}}$ are consequences of the old edge and conjugation relations. By taking the barycentric subdivision, we can assure that $X / G$ is the simplicial complex whose simplices are the orbits of simplices in $X$. 
Remark If $G$ did not act without rotations, then we would need to take the second barycentric subdivision to assure that $X / G$ is the simplicial complex whose simplices are the orbits of simplices in $X$.

Let $\pi: X \rightarrow X / G$ be the projection and let

$$
\Gamma=\left(\underset{x \in X^{(0)}}{*} G_{x}\right) / R
$$

be as in the statement of the theorem. As in the introduction, for $x \in X^{(0)}$ and $h \in G_{X}$, we will denote by $h_{x} \in \Gamma$ the corresponding element of $G_{x}<\Gamma$. There is an obvious homomorphism $\psi: \Gamma \rightarrow G$. We will construct a surjective homomorphism $\psi^{-1}: G \rightarrow \Gamma$ such that $\psi \circ \psi^{-1}=1$; the theorem will immediately follow.

The proof will have three parts. In Section 3.1, we give a procedure (due to Armstrong [1]) for expressing an element of $G$ as a product of vertex stabilizers. This procedure is reminiscent of standard arguments involving covering spaces. In Section 3.2, we will show that the resulting element of $\Gamma$ is independent of the choices made. This will define $\psi^{-1}$. Finally, in Section 3.3 we will show that $\psi^{-1}$ is a surjective homomorphism.

Throughout the whole proof, we will fix some basepoint $\widetilde{v} \in X^{(0)}$ and define $v=$ $\pi(\widetilde{v}) \in X / G$.

\subsection{Expressing elements of $G$ as products of vertex stabilizers: the Arm- strong construction}

Consider $g \in G$. Let $\tilde{\gamma}$ be a simplicial path in $X$ from $\tilde{v}$ to $g(\widetilde{v})$. The projection $\pi$ sends $\tilde{\gamma}$ to a closed loop in $X / G$ based at $v$. Since $X / G$ is simply-connected, there is some simplicial map $\phi:(D, *) \rightarrow(X / G, v)$, where $(D, \gamma)$ is a nondegenerate disc and $\phi_{*}(\gamma)=\pi_{*}(\tilde{\gamma})$ (in fact, for later use we point out that in our construction, we will never use the nondegeneracy of $(D, \gamma))$. This is all illustrated in Figure 2(a).

Since $(D, \gamma)$ is a degenerate disc, Corollary 2.5 says that there is a sequence

$$
(D, \gamma)=\left(D_{1}, \gamma_{1}\right),\left(D_{2}, \gamma_{2}\right), \ldots,\left(D_{n}, \gamma_{n}\right)=(*, *)
$$

of degenerate discs such that for $1 \leq i<n$, the degenerate disc $\left(D_{i+1}, \gamma_{i+1}\right)$ differs from $\left(D_{i}, \gamma_{i}\right)$ by either a one- or two-dimensional collapse. Each $D_{i}$ is a subcomplex of $D$, so $\phi$ restricts to a map $\phi_{i}: D_{i} \rightarrow X / G$. The resulting sequence of based loops $\left(\phi_{i}\right)_{*}\left(\gamma_{i}\right)$ gives a simplicial homotopy from $\pi_{*}(\tilde{\gamma})$ to the constant path. We will construct a sequence of paths $\tilde{\gamma}_{1}, \ldots, \tilde{\gamma}_{n}$ in $X$ which all start at $v$ and which satisfy $\pi_{*}\left(\tilde{\gamma}_{i}\right)=\left(\phi_{i}\right)_{*}\left(\gamma_{i}\right)$ for $1 \leq i \leq n$. Additionally, our construction will yield a sequence 
$\widetilde{v}_{1}, \ldots, \widetilde{v}_{n}$ of vertices of $X$ and a sequence $h_{1}, \ldots, h_{n}$ of elements of $G$ such that $h_{i} \in G_{\widetilde{v}_{i}}$ for $1 \leq i \leq n$ and such that $h_{1}^{-1} \cdots h_{n}^{-1}=g$.

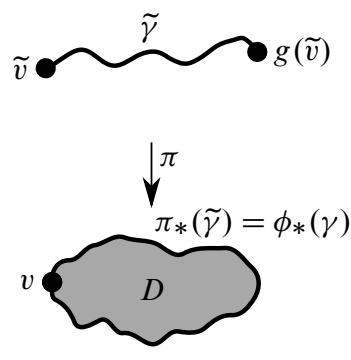

(a)

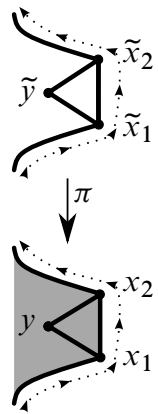

(b)

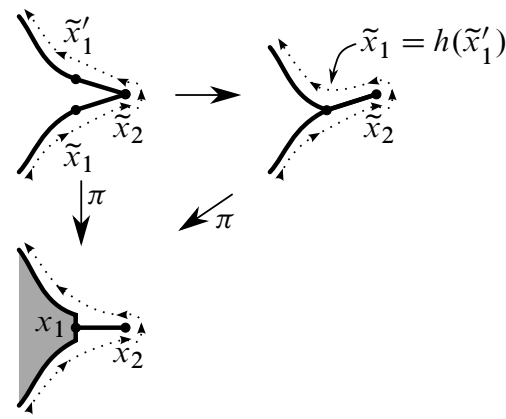

(c)

Figure 2: (a) $\tilde{\gamma}$ and its projection (b) Lifting a two-dimensional collapse (c) Swinging around a pivot point to lift a one-dimensional collapse

Begin by defining $\tilde{\gamma}_{1}=\tilde{\gamma}$. Assume that for some $1 \leq k<n$ we have constructed paths $\tilde{\gamma}_{1}, \ldots, \tilde{\gamma}_{k}$ with the indicated properties. If $\left(D_{k+1}, \gamma_{k+1}\right)$ differs from $\left(D_{k}, \gamma_{k}\right)$ by a two-dimensional collapse, then by definition there is some simplex $\left\{x_{1}, x_{2}, y\right\}$ of $D_{k}$ such that $x_{1}-x_{2}$ is a subpath of $\gamma_{k}$ which is homotoped to the subpath $x_{1}-y-x_{2}$ of $\gamma_{k+1}$ (see the bottom portion of Figure 2(b)). Let $\tilde{x}_{1}-\tilde{x}_{2}$ be the relevant portion of $\tilde{\gamma}$. Since simplices in $X / G$ are exactly the orbits of simplices in $X$, there exists some $\tilde{y} \in X^{(0)}$ such that $\pi(\tilde{y})=\phi_{k}(y)$ and such that $\left\{\tilde{x}_{1}, \tilde{x}_{2}, \tilde{y}\right\}$ is a simplex of $X$ (see the top portion of Figure 2(b); we wish to point out that since $\phi_{k}$ need not be injective, we might have $\tilde{y}=\tilde{x}_{i}$ for some $i$ ). Let $\tilde{\gamma}_{k+1}$ equal $\tilde{\gamma}_{k}$ with the subpath $\tilde{x}_{1}-\tilde{x}_{2}$ replaced with $\tilde{x}_{1}-\tilde{y}-\tilde{x}_{2}$. It is clear that $\tilde{\gamma}_{k+1}$ projects to $\left(\phi_{k+1}\right)_{*}\left(\gamma_{k+1}\right)$. In this case, we set $\widetilde{v}_{k}=\tilde{v}$ and $h_{k}=1$.

Remark Since $h_{k}=1$, the precise value of $\tilde{v}_{k}$ is immaterial.

If $\left(D_{k+1}, \gamma_{k+1}\right)$ differs from $\left(D_{k}, \gamma_{k}\right)$ by a one-dimensional collapse, then we may have to modify $\tilde{\gamma}_{k}$ before homotoping it. Indeed, let $x_{1}-x_{2}-x_{1}$ be the relevant subpath of $\gamma_{k}$. As indicated in Figure 2(c), the portion of $\tilde{\gamma}_{k}$ which projects to $\phi_{k}\left(x_{1}\right)-\phi_{k}\left(x_{2}\right)-\phi_{k}\left(x_{1}\right)$ may be of the form $\tilde{x}_{1}-\tilde{x}_{2}-\tilde{x}_{1}^{\prime}$ with $\pi\left(\tilde{x}_{1}\right)=\pi\left(\tilde{x}_{1}^{\prime}\right)$ but with $\tilde{x}_{1} \neq \tilde{x}_{1}^{\prime}$ (observe that if this happens, then we must have $\phi_{k}\left(x_{1}\right) \neq \phi_{k}\left(x_{2}\right)$ ). However, since simplices of $X / G$ are the orbits of simplices in $X$, there must exist some $h \in G_{\tilde{x}_{2}}$ such that $h_{k}\left(\tilde{x}_{1}^{\prime}\right)=\tilde{x}_{1}$ (if $\tilde{x}_{1}^{\prime}=\tilde{x}_{1}$, then $h=1$ ). Let $\rho_{1}$ be the portion of $\tilde{\gamma}_{k}$ before $\tilde{x}_{1}-\tilde{x}_{2}-\tilde{x}_{1}^{\prime}$ and $\rho_{2}$ be the portion after it. We then define $\tilde{\gamma}_{k+1}^{\prime}$ to equal

$$
\rho_{1}-\tilde{x}_{1}-\tilde{x}_{2}-\tilde{x}_{1}-h_{k}\left(\rho_{2}\right)
$$


see the top right hand portion of Figure 2(c). We will call this the result of swinging $\tilde{\gamma}_{k}$ around the pivot point $\tilde{x}_{2}$ by $h_{k}$. We then can remove $\tilde{x}_{2}$ from $\tilde{\gamma}_{k+1}^{\prime}$ to get $\tilde{\gamma}_{k+1}$, a lift of $\left(\phi_{k+1}\right)_{*}\left(\gamma_{k+1}\right)$. Define $\widetilde{v}_{k}=\tilde{x}_{2}$ and $h_{k}=h$.

Remark Technically speaking, our pivot point $\tilde{x}_{2}$ lies in $X$, not on $\tilde{\gamma}$, and in fact $\tilde{\gamma}$ may pass through $\tilde{x}_{2}$ multiple times. However, to simplify our notation we will abuse notation and always assume that pivot points correspond to specific points on our paths. In particular, if we say that two pivot points on a path are the same, we mean not merely that they are the same point of $X$ but that moreover they correspond to the same point on the path.

We have now constructed the paths $\tilde{\gamma}_{1}, \ldots, \tilde{\gamma}_{n}$, the vertices $\tilde{v}_{1}, \ldots, \widetilde{v}_{n-1}$, and the group elements $h_{1}, \ldots, h_{n-1}$ with $h_{i} \in G_{\widetilde{v}_{i}}$ for $1 \leq i \leq n-1$. For $1 \leq i<n$, it is immediate from our construction that the endpoint of $\tilde{\gamma}_{i+1}$ is equal to the result of applying $h_{i}$ to the endpoint of $\tilde{\gamma}_{i}$. Since the endpoint of $\tilde{\gamma}_{1}$ is $g(\tilde{v})$, we deduce that the endpoint of $\tilde{\gamma}_{n}$ is $h_{n-1} \cdots h_{1} \cdot g(\widetilde{v})$. But $\tilde{\gamma}_{n}$ is the constant path $\widetilde{v}$. We conclude that

$$
h_{n-1} \cdots h_{1} \cdot g(\widetilde{v})=\tilde{v} .
$$

Define $\widetilde{v}_{n}=\tilde{v}$ and $h_{n}=\left(h_{n-1} \cdots h_{1} \cdot g\right)^{-1}$. We thus have $h_{n} \in G_{\widetilde{v}_{n}}$ and

$$
g=h_{1}^{-1} \cdots h_{n}^{-1}
$$

as desired.

Observe that

$$
\psi\left(\left(h_{1}\right)_{\tilde{v}_{1}}^{-1} \cdots\left(h_{n}\right)_{\widetilde{v}_{n}}^{-1}\right)=g
$$

We will say that

$$
\left(h_{1}\right)_{\widetilde{v}_{1}}^{-1} \cdots\left(h_{n}\right)_{\widetilde{v}_{n}}^{-1} \in \Gamma
$$

is the result of applying the Armstrong construction to $g$. Though a priori the output of the Armstrong construction depends on numerous choices, in Section 3.2 we will show that it is in fact independent of those choices.

For later use, we now define some notation. A degenerate disc $\left(D^{\prime}, \gamma^{\prime}\right)$ together with a lift $\tilde{\gamma}^{\prime}$ to $X$ of the image of $\gamma^{\prime}$ under some (implied) map from $D^{\prime}$ to $X / G$ will be denoted by $\left(D^{\prime}, \gamma^{\prime}, \tilde{\gamma}^{\prime}\right)$. We will denote the transition from $\left(D^{\prime}, \gamma^{\prime}, \tilde{\gamma}^{\prime}\right)$ to $\left(D^{\prime \prime}, \gamma^{\prime \prime}, \tilde{\gamma}^{\prime \prime}\right)$ coming from a one- or two-dimensional collapse plus swinging $\tilde{\gamma}^{\prime}$ around the pivot point $\widetilde{v}^{\prime}$ by $h^{\prime} \in G_{\widetilde{x}^{\prime}}$ by

$$
\left(D^{\prime}, \gamma^{\prime}, \tilde{\gamma}^{\prime}\right) \stackrel{h^{\prime}, \widetilde{v}^{\prime}}{\longrightarrow}\left(D^{\prime \prime}, \gamma^{\prime \prime}, \tilde{\gamma}^{\prime \prime}\right)
$$


We will denote the sequence of moves given by the above construction by

$$
\begin{aligned}
\phi:(D, \gamma, \tilde{\gamma})=\left(D_{1}, \gamma_{1}, \tilde{\gamma}_{1}\right) \stackrel{h_{1}, \tilde{v}_{1}}{\longrightarrow}\left(D_{2}, \gamma_{2}, \tilde{\gamma}_{2}\right) \stackrel{h_{2}, \tilde{v}_{2}}{\longrightarrow} \cdots \\
\cdots \\
\cdots \stackrel{h_{n-1}, \tilde{v}_{n-1}}{\longrightarrow}\left(D_{n}, \gamma_{n}, \tilde{\gamma}_{n}\right)=(*, *, \tilde{v}) .
\end{aligned}
$$

Such a sequence ending with $(*, *, \widetilde{v})$ will be called a full sequence of moves for $g$. We will also discuss partial sequences of moves, which are defined in the obvious way. Finally, we define the stabilizer product of (3) to be the product

$$
\left(h_{1}\right){\overline{\widetilde{v}_{1}}}^{1} \cdots\left(h_{n-1}\right){\overline{\tilde{v}_{n-1}}}^{1} \cdot\left(\left(h_{n-1} \cdots h_{1} \cdot g\right)^{-1}\right) \overline{\widetilde{v}}^{1}=\left(h_{1}\right){\overline{\sigma_{1}}}^{1} \cdots\left(h_{n}\right){\overline{\widetilde{v}_{n}}}^{1} \in \Gamma .
$$

We will also discuss the stabilizer products of partial sequences of moves, which are again defined in the obvious way (of course, in the stabilizer product of a partial sequence of moves, the $h_{n}$ term is omitted).

\subsection{The dependence of the Armstrong construction on our choices}

Fix $g \in G$. The goal of this section is to show that the element of $\Gamma$ obtained by applying the Armstrong construction to $g$ is independent of all of our arbitrary choices. Examining the construction, we see that the following is a complete list of such choices.

(1) The path $\tilde{\gamma}$ from $\tilde{v}$ to $g(\tilde{v})$.

(2) The nondegenerate disc $(D, \gamma)$ and the map $\phi: D \rightarrow X / G$ with $\phi_{*}(\gamma)=\pi_{*}(\tilde{\gamma})$.

(3) The manner in which we collapsed $\gamma$ across $D$ to homotope it to the trivial loop.

(4) The vertices $\tilde{y}$ involved in lifting two-dimensional collapses.

(5) The group elements $h_{i}$ chosen during the liftings of one-dimensional collapses.

We will deal with each choice in turn.

Remark The construction also depends on the choice of basepoint $\tilde{v} \in X^{(0)}$, but that was fixed at the beginning so there is no need to show that the output does not depend on it. In fact, it is not hard to show that the output of the Armstrong does not depend on $\tilde{v} \in X^{(0)}$ either.

Remark (On the assumptions in the theorem) The assumption that $X / G$ is simply connected was used during the Armstrong construction. The assumption that $X$ is simply connected is used to show that the output of the Armstrong construction does not depend on choice 1 and the assumption that $X / G$ is 2 -connected is used to show that the output of the Armstrong construction does not depend on choice 2 . These assumptions are not used in the proof that the output of the Armstrong construction does not depend on choices 3-5. 


\subsubsection{The output of the Armstrong construction does not depend on choices 3-5} The proof will be by induction on the number $m$ of simplices (of any dimension) in the nondegenerate disc $D$. In fact, for use in our induction we will allow $(D, \gamma)$ to be a degenerate disc; as we observed in Section 3.1, the nondegeneracy of $(D, \gamma)$ was never used during the Armstrong construction.

The case $m=1$ is trivial; in that case, our expression for $\psi^{-1}(g)$ is simply $g_{\widetilde{v}}$. Assume, therefore, that $m>1$. Consider two possible first moves

$$
\phi:(D, \gamma, \tilde{\gamma}) \stackrel{h_{1}, \widetilde{v}_{1}}{\longrightarrow}\left(D_{2}, \gamma_{2}, \tilde{\gamma}_{2}\right)
$$

and $\quad \phi:(D, \gamma, \tilde{\gamma}) \stackrel{h_{1}^{\prime}, \widetilde{v}_{1}^{\prime}}{\longrightarrow}\left(D_{2}^{\prime}, \gamma_{2}^{\prime}, \tilde{\gamma}_{2}^{\prime}\right)$.

We will show that there exist full sequences of moves

$$
\begin{aligned}
& \phi:(D, \gamma, \tilde{\gamma}) \stackrel{h_{1}, \widetilde{v}_{1}}{\longrightarrow}\left(D_{2}, \gamma_{2}, \tilde{\gamma}_{2}\right) \rightarrow \cdots \\
& \text { and } \quad \phi:(D, \gamma, \tilde{\gamma}) \stackrel{h_{1}^{\prime}, \widetilde{v}_{1}^{\prime}}{\longrightarrow}\left(D_{2}^{\prime}, \gamma_{2}^{\prime}, \widetilde{\gamma}_{2}^{\prime}\right) \rightarrow \cdots
\end{aligned}
$$

such that the stabilizer products of (6) and (7) are the same lift of $g$ to $\Gamma$. This is enough to prove the claim. Indeed, let

$$
\begin{aligned}
& \phi:(D, \gamma, \tilde{\gamma}) \stackrel{h_{1}, \widetilde{v}_{1}}{\longrightarrow}\left(D_{2}, \gamma_{2}, \tilde{\gamma}_{2}\right) \rightarrow \cdots \\
& \text { and } \quad \phi:(D, \gamma, \tilde{\gamma}) \stackrel{h_{1}^{\prime}, \widetilde{v}_{1}^{\prime}}{\longrightarrow}\left(D_{2}^{\prime}, \gamma_{2}^{\prime}, \widetilde{\gamma}_{2}^{\prime}\right) \rightarrow \cdots
\end{aligned}
$$

be two arbitrary full sequences of moves across $D$ starting with (4) and (5). The subsequences of moves obtained by deleting the initial $(D, \gamma, \tilde{\gamma})$ from (6) and (8) yield full sequences of moves across $D_{2}$. The stabilizer products of these subsequences give two lifts to $\Gamma$ of $h_{1} g$, and by our inductive hypothesis these two elements of $\Gamma$ are equal. Thus the stabilizer products of (6) and (8) are the same lift of $g$ to $\Gamma$. Similarly, the stabilizer products of (7) and (9) are the same lift of $g$ to $\Gamma$. We conclude that the stabilizer products of (8) and (9) are the same lift of $g$ to $\Gamma$, as desired.

The heart of the proof will be the following three special cases.

Case 1 The simplices of $D$ deleted in (4) and (5) are distinct.

Case $2 D$ is a line

Case $3 D$ is a flower whose bloom consists of a single simplex. 
These cases are enough to prove the claim. Indeed, assume that we are not in Case 1, so the simplices of $D$ deleted in (4) and (5) are identical. If there is some other first move

$$
\phi:(D, \gamma, \tilde{\gamma}) \stackrel{h_{1}^{\prime \prime}, \widetilde{v}_{1}^{\prime \prime}}{\longrightarrow}\left(D_{2}^{\prime \prime}, \gamma_{2}^{\prime \prime}, \tilde{\gamma}_{2}^{\prime \prime}\right)
$$

which deletes a different simplex, then Case 1 applies to (10) together with either (4) or (5). Thus there exist full sequences of moves

$$
\begin{aligned}
& \phi:(D, \gamma, \tilde{\gamma}) \stackrel{h_{1}, \widetilde{v}_{1}}{\longrightarrow}\left(D_{2}, \gamma_{2}, \tilde{\gamma}_{2}\right) \rightarrow \cdots \\
& \phi:(D, \gamma, \tilde{\gamma}) \stackrel{h_{1}^{\prime \prime}, \widetilde{v}_{1}^{\prime \prime}}{\longrightarrow}\left(D_{2}^{\prime \prime}, \gamma_{2}^{\prime \prime}, \widetilde{\gamma}_{2}^{\prime \prime}\right) \rightarrow \cdots \\
& \phi:(D, \gamma, \tilde{\gamma}) \stackrel{h_{1}^{\prime}, \widetilde{v}_{1}^{\prime}}{\longrightarrow}\left(D_{2}^{\prime}, \gamma_{2}^{\prime}, \widetilde{\gamma}_{2}^{\prime}\right) \rightarrow \cdots \\
& \phi:(D, \gamma, \tilde{\gamma}) \stackrel{h_{1}^{\prime \prime}, \widetilde{v}_{1}^{\prime \prime}}{\longrightarrow}\left(D_{2}^{\prime \prime}, \gamma_{2}^{\prime \prime}, \widetilde{\gamma}_{2}^{\prime \prime}\right) \rightarrow \cdots
\end{aligned}
$$

such that the stabilizer products of (11) and (12) (resp. (13) and (14)) are the same. Using the inductive hypothesis like we did above, the stabilizer products of (12) and (14) are the same. We conclude that the stabilizer products of (11) and (13) are the same, as desired.

If instead there is no move deleting a different simplex, then using Corollary 2.7 we can conclude that $D$ is a line (if (4) and (5) are one-dimensional collapses) or a flower whose bloom consists of a single simplex (if (4) and (5) are two-dimensional collapses). We can thus apply either Case 2 or Case 3, and we are done.

The proofs of all three cases follow the same pattern. Namely, for some $N \geq 2$ we construct partial sequences of moves

$$
\phi:(D, \gamma, \tilde{\gamma}) \stackrel{h_{1}, \widetilde{v}_{1}}{\longrightarrow}\left(D_{2}, \gamma_{2}, \tilde{\gamma}_{2}\right) \stackrel{h_{2}, \widetilde{v}_{2}}{\longrightarrow} \cdots \stackrel{h_{N-1}, \widetilde{v}_{N-1}}{\longrightarrow}\left(D_{N}, \gamma_{N}, \tilde{\gamma}_{N}\right)
$$

(16) and

$$
\phi:(D, \gamma, \tilde{\gamma}) \stackrel{h_{1}^{\prime}, \widetilde{v}_{1}^{\prime}}{\longrightarrow}\left(D_{2}^{\prime}, \gamma_{2}^{\prime}, \tilde{\gamma}_{2}^{\prime}\right) \stackrel{h_{2}^{\prime}, \widetilde{v}_{2}^{\prime}}{\longrightarrow} \cdots \stackrel{h_{N^{\prime}-1}^{\prime}, \widetilde{v}_{N^{\prime}-1}^{\prime}}{\longrightarrow}\left(D_{N^{\prime}}^{\prime}, \gamma_{N^{\prime}}^{\prime}, \widetilde{\gamma}_{N^{\prime}}^{\prime}\right)
$$

with $\left(D_{N}, \gamma_{N}, \tilde{\gamma}_{N}\right)=\left(D_{N^{\prime}}^{\prime}, \gamma_{N^{\prime}}^{\prime}, \tilde{\gamma}_{N^{\prime}}^{\prime}\right)$. We then verify that the stabilizer products of (15) and (16) are equal; that is, that

$$
\left(h_{1}\right)_{\widetilde{v}_{1}}^{-1} \cdots\left(h_{N-1}\right)_{\tilde{v}_{N-1}}^{-1}=\left(h_{1}^{\prime}\right)_{\widetilde{v}_{1}^{\prime}}^{-1} \cdots\left(h_{N^{\prime}-1}^{\prime}\right)_{\widetilde{v}_{N^{\prime}-1}}^{-1} .
$$

It will then follow that the desired full sequences of moves can be obtained by completing (15) and (16) to full sequences of moves in the same way.

Proof of Case 1 (distinct simplices deleted) There are three cases. 


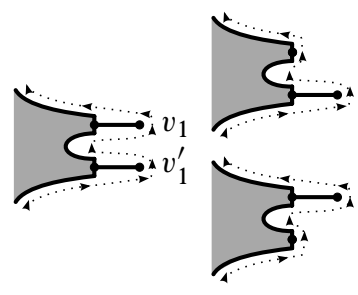

(a)

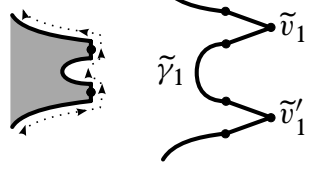

(b)
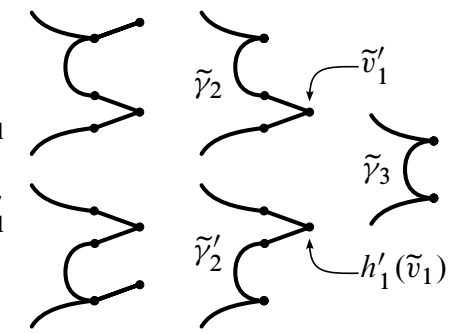

b)

Figure 3: (a) Left is $\left(D_{1}, \gamma_{1}\right)$, middle top is $\left(D_{2}, \gamma_{2}\right)$, middle bottom is $\left(D_{2}^{\prime}, \gamma_{2}^{\prime}\right)$, right is $\left(D_{3}, \gamma_{3}\right)$ (b) The various lifts needed

(1) Both (4) and (5) correspond to two-dimensional collapses.

(2) Both (4) and (5) correspond to one-dimensional collapses.

(3) (4) and (5) correspond to different types of collapses.

The argument is similar in all three cases; we will discuss the slightly more difficult case 2 and leave cases 1 and 3 to the reader.

Assume, therefore, that (4) and (5) correspond to one-dimensional collapses. The proof is illustrated in Figure 3. Let $v_{1}$ and $v_{1}^{\prime}$ be the vertices of $\gamma$ which map to $\pi\left(\widetilde{v}_{1}\right)$ and $\pi\left(\widetilde{v}_{1}^{\prime}\right)$. Without loss of generality, we can assume that $v_{1}^{\prime}$ comes before $v_{1}$ in $\gamma$ (see Figure 3(a)). Let $\left(D_{3}, \gamma_{3}\right)$ be the result of performing a one-dimensional collapse to $\left(D_{2}, \gamma_{2}\right)$ at $v_{1}^{\prime}$, or equivalently the result of performing a one-dimensional collapse to $\left(D_{2}^{\prime}, \gamma_{2}\right)$ at $v_{1}$ (see Figure 3(a)).

Observe that swinging $\tilde{\gamma}$ around $\widetilde{v}_{1}$ by $h_{1}$ does not affect $\widetilde{v}_{1}^{\prime}$, so we can lift $\left(\phi_{3}\right)_{*}\left(\gamma_{3}\right)$ to a path $\tilde{\gamma}_{3}$ in $X$ by swinging $\tilde{\gamma}_{2}$ around $\tilde{v}_{1}^{\prime}$ by $h_{1}^{\prime}$ and then removing $\widetilde{v}_{1}^{\prime}$ from $\tilde{\gamma}_{2}$ (see Figure $3(\mathrm{~b})$ ). Similarly, swinging $\tilde{\gamma}$ around $\widetilde{v}_{1}^{\prime}$ by $h_{1}^{\prime}$ moves $\widetilde{v}_{1}$ to $h_{1}^{\prime}\left(\widetilde{v}_{1}\right)$, so we can lift $\left(\phi_{3}\right)_{*}\left(\gamma_{3}\right)$ to $X$ by swinging $\tilde{\gamma}_{2}^{\prime}$ around $h_{1}^{\prime}\left(\widetilde{v}_{1}\right)$ by $h_{1}^{\prime} h_{1}\left(h_{1}^{\prime}\right)^{-1}$ and then removing $h_{1}^{\prime}\left(\widetilde{v}_{1}\right)$; the resulting path is equal to $\widetilde{\gamma}_{3}$ (see Figure $3(\mathrm{~b})$ ).

We thus have constructed sequences of moves

$$
\phi:(D, \gamma, \tilde{\gamma}) \stackrel{h_{1}, \widetilde{v}_{1}}{\longrightarrow}\left(D_{2}, \gamma_{2}, \tilde{\gamma}_{2}\right) \stackrel{h_{1}^{\prime}, \tilde{v}_{1}^{\prime}}{\longrightarrow}\left(D_{3}, \gamma_{3}, \tilde{\gamma}_{3}\right)
$$

and $\quad \phi:(D, \gamma, \tilde{\gamma}) \stackrel{h_{1}^{\prime}, \widetilde{v}_{1}^{\prime}}{\longrightarrow}\left(D_{2}^{\prime}, \gamma_{2}^{\prime}, \widetilde{\gamma}_{2}^{\prime}\right) \stackrel{h_{1}^{\prime} h_{1}\left(h_{1}^{\prime}\right)^{-1}, h_{1}^{\prime}\left(\widetilde{v}_{1}\right)}{\longrightarrow}\left(D_{3}, \gamma_{3}, \widetilde{\gamma}_{3}\right)$.

In $\Gamma$ we have

$$
\left(h_{1}\right)_{\widetilde{v}_{1}}^{-1}\left(h_{1}^{\prime}\right)_{\widetilde{v}_{1}^{\prime}}^{-1}=\left(h_{1}^{\prime}\right)_{\widetilde{v}_{1}^{\prime}}^{-1}\left(h_{1}^{\prime}\right)_{\widetilde{v}_{1}^{\prime}}\left(h_{1}\right)_{\widetilde{v}_{1}}^{-1}\left(h_{1}^{\prime}\right)_{\widetilde{v}_{1}^{\prime}}^{-1}=\left(h_{1}^{\prime}\right)_{\widetilde{v}_{1}^{\prime}}^{-1}\left(h_{1}^{\prime} h_{1}\left(h_{1}^{\prime}\right)^{-1}\right)_{h_{1}^{\prime}\left(\widetilde{v}_{1}\right)},
$$

and the claim follows. 
Proof of Case 2 ( $D$ a line) This proof is illustrated in Figure 4 . We will do the case that $D$ has at least $k$ vertices for $k \geq 3$. The case that $D$ has 2 vertices (remember, it has more than 1) is similar, the key difference being that instead of the final "swinging" which occurs in our construction, use is made of the final element (denoted $h_{n}$ in Section 3.1) in the stabilizer product of a full sequence of moves.

Let the last three vertices of our line be $w_{1}, w_{2}$, and $w_{3}$ (see Figure 4(a)). As depicted in Figure 4(b), let the lift of the segment

$$
\phi\left(w_{1}\right)-\phi\left(w_{2}\right)-\phi\left(w_{3}\right)-\phi\left(w_{2}\right)-\phi\left(w_{1}\right)
$$

of $\phi_{*}(\gamma)$ to $\tilde{\gamma}$ be

$$
\widetilde{w}_{1}-\tilde{w}_{2}-\tilde{w}_{3}-\widetilde{w}_{2}^{\prime}-\widetilde{w}_{1}^{\prime}
$$

Observe that in (4) and (5), we must have $v_{1}=v_{1}^{\prime}=\tilde{w}_{3}$. We therefore have $\left(D_{2}, \gamma_{2}\right)=$ $\left(D_{2}^{\prime}, \gamma_{2}^{\prime}\right)$ (see Figure 4(a)). Let $\left(D_{3}, \gamma_{3}\right)$ be as in Figure 4(a).

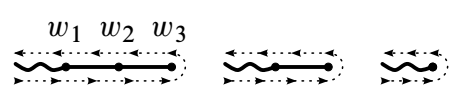

(a)

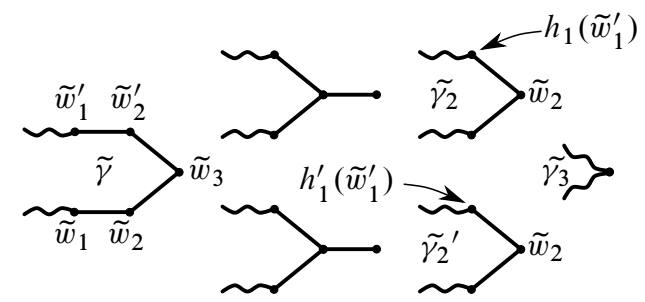

(b)

Figure 4: (a) Left is $(D, \gamma)$, middle is $\left(D_{2}, \gamma_{2}\right)=\left(D_{2}^{\prime}, \gamma_{2}^{\prime}\right)$, right is $\left(D_{3}, \gamma_{3}\right)$ (b) Lifts to $X$

Next, observe that

$$
h_{1}\left(\tilde{w}_{2}^{\prime}\right)=h_{1}^{\prime}\left(\tilde{w}_{2}^{\prime}\right)=\tilde{w}_{2} .
$$

This implies that $h_{1}^{\prime}=\mu h_{1}$ for some $\mu \in G_{\left\{\tilde{w}_{3}, \tilde{w}_{2}\right\}}$. Observe that $\tilde{\gamma}_{2}$ and $\tilde{\gamma}_{2}^{\prime}$ are as depicted in Figure 4(b). Let $h_{2} \in G_{\widetilde{w}_{2}}$ be such that

$$
h_{2}\left(h_{1}\left(\widetilde{w}_{1}^{\prime}\right)\right)=\widetilde{w}_{1} .
$$

We can therefore obtain a lift $\tilde{\gamma}_{3}$ of $\phi_{*}\left(\gamma_{3}\right)$ to $X$ by swinging $\tilde{\gamma}_{2}$ around the pivot point $\tilde{w}_{2}$ by $h_{2}$ and then deleting $\tilde{w}_{2}$ from it. We thus have a sequence of moves

$$
\phi:(D, \gamma, \tilde{\gamma}) \stackrel{h_{1}, \widetilde{w}_{3}}{\longrightarrow}\left(D_{2}, \gamma_{2}, \tilde{\gamma}_{2}\right) \stackrel{h_{2}, \widetilde{w}_{2}}{\longrightarrow}\left(D_{3}, \gamma_{3}, \tilde{\gamma}_{3}\right)
$$

as depicted in Figure 4(b).

Since $\mu, h_{2} \in G_{\widetilde{w}_{2}}$, it follows that $h_{2} \mu^{-1} \in G_{\widetilde{w}_{2}}$. Also, we have

$$
\left(h_{2} \mu^{-1}\right)\left(h_{1}^{\prime}\left(\widetilde{w}_{1}^{\prime}\right)\right)=h_{2} \mu^{-1} \mu h_{1}\left(\widetilde{w}_{1}^{\prime}\right)=h_{2}\left(h_{1}\left(\widetilde{w}_{1}^{\prime}\right)\right)=\widetilde{w}_{1} .
$$


We can thus obtain a lift $\tilde{\gamma}_{3}^{\prime}$ of $\phi_{*}\left(\gamma_{3}^{\prime}\right)$ to $X$ by swinging $\tilde{\gamma}_{2}^{\prime}$ around the pivot point $\tilde{w}_{2}$ by $h_{2} \mu^{-1}$ and then deleting $\tilde{w}_{2}$ from it. Moreover, it is clear that $\tilde{\gamma}_{3}^{\prime}=\tilde{\gamma}_{3}$. We thus have a sequence of moves

$$
\phi:(D, \gamma, \tilde{\gamma}) \stackrel{h_{1}^{\prime}, \widetilde{w}_{3}}{\longrightarrow}\left(D_{2}^{\prime}, \gamma_{2}^{\prime}, \tilde{\gamma}_{2}^{\prime}\right) \stackrel{h_{2} \mu^{-1}, \widetilde{w}_{2}}{\longrightarrow}\left(D_{3}, \gamma_{3}, \tilde{\gamma}_{3}\right)
$$

Since in $\Gamma$ we have

$$
\begin{aligned}
\left(h_{1}^{\prime}\right)_{\widetilde{w}_{3}}^{-1}\left(h_{2} \mu^{-1}\right)_{\widetilde{w}_{2}}^{-1} & =\left(h_{1}\right)_{\widetilde{w}_{3}}^{-1}(\mu)_{\widetilde{w}_{3}}^{-1}(\mu)_{\widetilde{w}_{2}}\left(h_{2}\right)_{\widetilde{w}_{2}}^{-1} \\
& =\left(h_{1}\right)_{\widetilde{w}_{3}}^{-1}(\mu)_{\widetilde{w}_{3}}^{-1}(\mu) \widetilde{w}_{3}\left(h_{2}\right)_{\widetilde{w}_{2}}^{-1} \\
& =\left(h_{1}\right)_{\widetilde{w}_{3}}^{-1}\left(h_{2}\right)_{\widetilde{w}_{2}}^{-1},
\end{aligned}
$$

the claim follows.

Proof of Case 3 ( $D$ a flower) We will assume that the stem of our flower has at least two vertices (that is, there is more to the flower than the bloom - the bloom and the stem share one vertex); the case where the stem consists of a single vertex is similar. As in the top portion of Figure 5(a), let $w_{1}-w_{2}$ be the last edge of the stem of $D$ and let the path around the bloom of $D$ be $w_{2}-x-y-w_{2}$. There are two cases.

Subcase 1 The edges deleted by (4) and (5) are equal.
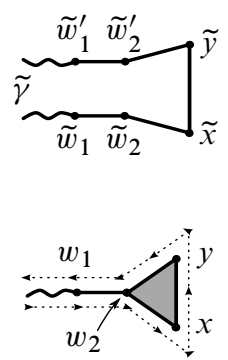

(a)
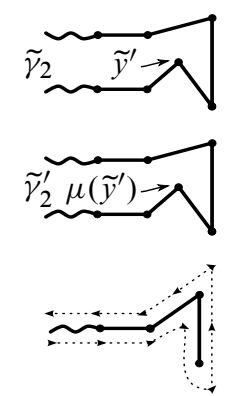

(b)

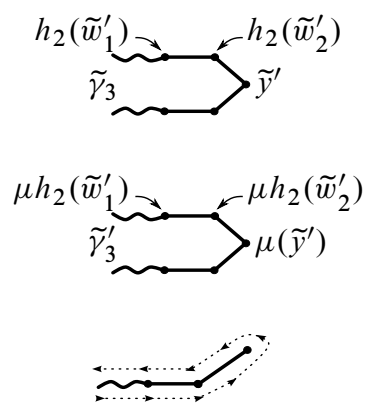

(c)
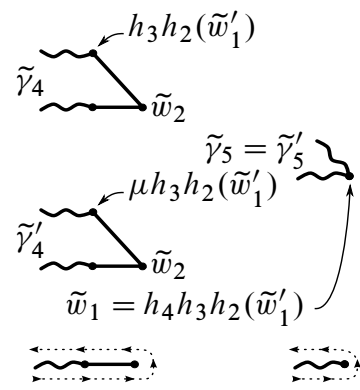

(d)

Figure 5: (a) Bottom is $(D, \gamma)$, top is $\tilde{\gamma}$ (b)-(e) Bottoms are $\left(D_{2}, \gamma_{2}\right)=$ $\left(D_{2}^{\prime}, \gamma_{2}^{\prime}\right)$ through $\left(D_{5}, \gamma_{5}\right)=\left(D_{5}^{\prime}, \gamma_{5}^{\prime}\right)$, tops are lifts to $X$

This is similar to the case of the line, so our exposition will be slightly terse. We will do the case that (4) and (5) delete the edge $w_{2}-x$; the other two cases are similar. Observe that $\left(D_{2}, \gamma_{2}\right)=\left(D_{2}^{\prime}, \gamma_{2}^{\prime}\right)$ and that $\left(D_{2}, \gamma_{2}\right)$ is as depicted on the bottom of Figure 5(b). Also, since (4) and (5) are both two-dimensional collapses, it follows that $h_{1}=h_{1}^{\prime}=1$. Let $\left(D_{i}, \gamma_{i}\right)$ for $3 \leq i \leq 5$ be as in the bottom portions of Figures 5(c)-(e). 
Let the portion of $\tilde{\gamma}_{1}$ corresponding to the last two vertices of the stem plus the path around the bloom be

$$
\widetilde{w}_{1}-\widetilde{w}_{2}-\tilde{x}-\tilde{y}-\widetilde{w}_{2}^{\prime}-\widetilde{w}_{1}^{\prime}
$$

and let the corresponding portion of $\tilde{\gamma}_{2}$ be

$$
\widetilde{w}_{1}-\widetilde{w}_{2}-\tilde{y}^{\prime}-\tilde{x}-\tilde{y}-\widetilde{w}_{2}^{\prime}-\widetilde{w}_{1}^{\prime} ;
$$

see the tops of Figures 5(a),(b). Then as shown in Figures 5(a)-(e), there exists $h_{2} \in G_{\tilde{x}}$, $h_{3} \in G_{\widetilde{y}^{\prime}}$, and $h_{4} \in G_{\widetilde{w}_{2}}$ such that we have a sequence of moves

$\phi:(D, \gamma, \tilde{\gamma}) \stackrel{h_{1}, \widetilde{v}_{1}}{\longrightarrow}\left(D_{2}, \gamma_{2}, \tilde{\gamma}_{2}\right) \stackrel{h_{2}, \tilde{x}}{\longrightarrow}\left(D_{3}, \gamma_{3}, \tilde{\gamma}_{3}\right) \stackrel{h_{3}, \tilde{y}^{\prime}}{\longrightarrow}\left(D_{4}, \gamma_{4}, \tilde{\gamma}_{4}\right) \stackrel{h_{4}, \widetilde{w}_{2}}{\longrightarrow}\left(D_{5}, \gamma_{5}, \tilde{\gamma}_{5}\right)$.

Moreover, as in the case of the line, there must exist some $\mu \in G_{\left\{\tilde{w}_{2}, \tilde{x}\right\}}$ such that the lift of the portion of $\tilde{\gamma}_{2}^{\prime}$ corresponding to (19) is

$$
\tilde{w}_{1}-\tilde{w}_{2}-\mu\left(\tilde{y}^{\prime}\right)-\tilde{x}-\tilde{y}-\tilde{w}_{2}^{\prime}-\widetilde{w}_{1}^{\prime} .
$$

Sine $\mu \in G_{\left\{\tilde{w}_{2}, \tilde{x}\right\}}$, we have $\mu h_{2} \in G_{\tilde{x}}$ and $h_{4} \mu^{-1} \in G_{\widetilde{w}_{2}}$. Thus as shown in Figures $5(\mathrm{a})-(\mathrm{e})$, there exists a sequence of moves

$\phi:(D, \gamma, \tilde{\gamma}) \stackrel{h_{1}^{\prime}, \widetilde{v}_{1}^{\prime}}{\longrightarrow}\left(D_{2}^{\prime}, \gamma_{2}^{\prime}, \tilde{\gamma}_{2}^{\prime}\right) \stackrel{\mu h_{2}, \tilde{x}}{\longrightarrow}\left(D_{3}^{\prime}, \gamma_{3}^{\prime}, \tilde{\gamma}_{3}^{\prime}\right)$

$$
\stackrel{\mu h_{3} \mu^{-1}, \mu\left(\tilde{y}^{\prime}\right)}{\longrightarrow}\left(D_{4}^{\prime}, \gamma_{4}^{\prime}, \tilde{\gamma}_{4}^{\prime}\right) \stackrel{h_{4} \mu^{-1}, \tilde{w}_{2}}{\longrightarrow}\left(D_{5}^{\prime}, \gamma_{5}^{\prime}, \tilde{\gamma}_{5}^{\prime}\right) \text {. }
$$

Also, we have $\left(D_{5}^{\prime}, \gamma_{5}^{\prime}, \tilde{\gamma}_{5}^{\prime}\right)=\left(D_{5}, \gamma_{5}, \tilde{\gamma}_{5}\right)$. As we observed above, we have $h_{1}=$ $h_{1}^{\prime}=1$, so the proof is completed by observing that in $\Gamma$ we have

$$
\begin{aligned}
\left(\mu h_{2}\right)_{\tilde{x}}^{-1}\left(\mu h_{3} \mu^{-1}\right)_{\mu\left(\widetilde{y}^{\prime}\right)}^{-1}\left(h_{4} \mu^{-1}\right)_{\widetilde{w}_{2}}^{-1} & =\left(h_{2}\right)_{\tilde{x}}^{-1}(\mu)_{\tilde{x}}^{-1}(\mu)_{\tilde{x}}\left(h_{3}\right)_{\tilde{y}^{\prime}}^{-1}(\mu)_{\tilde{x}}^{-1}(\mu)_{\tilde{w}_{2}}\left(h_{4}\right)_{\tilde{w}_{2}}^{-1} \\
& =\left(h_{2}\right)_{\tilde{x}}^{-1}\left(h_{3}\right)_{\widetilde{y}^{\prime}}^{-1}(\mu)_{\tilde{x}}^{-1}(\mu)_{\tilde{x}}\left(h_{4}\right)_{\widetilde{w}_{2}}^{-1} \\
& =\left(h_{2}\right)_{\tilde{x}}^{-1}\left(h_{3}\right)_{\widetilde{y}^{\prime}}^{-1}\left(h_{4}\right)_{\widetilde{w}_{2}}^{-1}
\end{aligned}
$$

Subcase 2 The edges deleted by (4) and (5) are different.
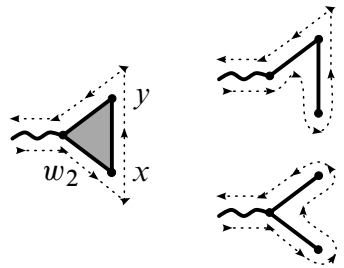

(a)
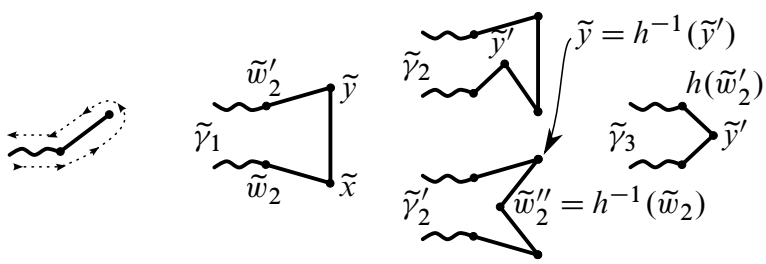

(b)

Figure 6: (a) Left is $\gamma$, middle top is $\gamma_{2}$, middle bottom is $\gamma_{2}^{\prime}$, right is $\gamma_{3}$ (b) Various lifts needed 
We will discuss the case that (4) deletes the edge $w_{2}-x$ and (5) deletes the edge $x-y$; the other cases are similar. Again, the details are similar to what we have already done, so we only sketch the argument.

Observe that $\left(D_{2}, \gamma_{2}\right)$ and $\left(D_{2}^{\prime}, \gamma_{2}^{\prime}\right)$ are as depicted in the top and bottom of the middle of Figure 6(a), respectively. Also, since (4) and (5) are both two-dimensional collapses, it follows that $h_{1}=h_{1}^{\prime}=1$. Let $\left(D_{3}, \gamma_{3}\right)$ be as in the right hand portion of Figure 6(a). As in Figure 6(b), let the portions of $\tilde{\gamma}_{2}$ and $\tilde{\gamma}_{2}^{\prime}$ corresponding to the last vertex of the stem plus the path around the bloom be

$$
\begin{array}{ll} 
& \tilde{w}_{2}-\tilde{y}^{\prime}-\tilde{x}-\tilde{y}-\widetilde{w}_{2}^{\prime} \\
\text { and } \quad & \tilde{w}_{2}-\tilde{x}-\tilde{w}_{2}^{\prime \prime}-\tilde{y}-\tilde{w}_{2}^{\prime},
\end{array}
$$

respectively (we will discuss the identities $\widetilde{w}_{2}^{\prime \prime}=h^{-1}\left(\widetilde{w}_{2}\right)$ and $\tilde{y}=h^{-1}\left(\widetilde{y}^{\prime}\right)$ in this figure shortly). Also, let

$$
\left.\phi\right|_{D_{2}}:\left(D_{2}, \gamma_{2}, \tilde{\gamma}_{2}\right) \stackrel{h_{2}, \tilde{x}}{\longrightarrow}\left(D_{3}, \gamma_{3}, \tilde{\gamma}_{3}\right)
$$

be an arbitrary lift of a one-dimensional collapse. Thus $h_{2} \in G_{\tilde{x}}$ and $h_{2}(\tilde{y})=\tilde{y}^{\prime}$. Using the previous case, we can modify $\widetilde{w}_{2}^{\prime \prime}$ to any legal vertex. In particular, we can assume that $\widetilde{w}_{2}^{\prime \prime}=h_{2}^{-1}\left(\widetilde{w}_{2}\right)$ (see Figure $6(\mathrm{~b})$ ). Thus

$$
\left.\phi\right|_{D_{2}^{\prime}}:\left(D_{2}^{\prime}, \gamma_{2}^{\prime}, \tilde{\gamma}_{2}^{\prime}\right) \stackrel{h_{2}, \tilde{x}}{\longrightarrow}\left(D_{3}, \gamma_{3}, \tilde{\gamma}_{2}\right)
$$

is a lift of a one-dimensional collapse. Summing up, we have sequences of moves

$$
\begin{aligned}
\phi:(D, \gamma, \tilde{\gamma}) & =\left(D_{1}, \gamma_{1}, \tilde{\gamma}_{1}\right) \stackrel{h_{1}, \tilde{v}_{1}}{\longrightarrow}\left(D_{2}, \gamma_{2}, \tilde{\gamma}_{2}\right) \stackrel{h_{2}, \tilde{x}}{\longrightarrow}\left(D_{3}, \gamma_{3}, \tilde{\gamma}_{3}\right) \\
\text { and } \quad \phi:(D, \gamma, \tilde{\gamma}) & =\left(D_{1}^{\prime}, \gamma_{1}^{\prime}, \tilde{\gamma}_{1}^{\prime}\right) \stackrel{h_{1}^{\prime}, \tilde{v}_{1}^{\prime}}{\longrightarrow}\left(D_{2}^{\prime}, \gamma_{2}^{\prime}, \tilde{\gamma}_{2}^{\prime}\right) \stackrel{h_{2}, \tilde{x}}{\longrightarrow}\left(D_{3}, \gamma_{3}, \tilde{\gamma}_{3}\right) .
\end{aligned}
$$

Since

$$
\left(h_{1}\right)_{\widetilde{v}_{1}}^{-1}\left(h_{2}\right)_{\tilde{x}}^{-1}=1 \cdot\left(h_{2}\right)_{\tilde{x}}^{-1}=\left(h_{1}^{\prime}\right)_{\widetilde{v}_{1}}^{-1}\left(h_{2}\right)_{\tilde{x}}^{-1},
$$

the result follows.

\subsubsection{The output of the Armstrong construction does not depend on choice 2 (the} degenerate disc $(D, \gamma))$. Recall that we have fixed some $g \in G$. By the previous section, the Armstrong construction associates a well-defined element of $\Gamma$ to the following data.

- A simplicial path $\tilde{\gamma}$ in $X$ from $\tilde{v}$ to $g(\tilde{v})$.

- A nondegenerate disc $(D, \gamma)$. 
- A simplicial map $\phi: D \rightarrow X / G$ such that $\phi_{*}(\gamma)=\pi_{*}(\tilde{\gamma})$.

In this section, we show that output of the Armstrong construction is independent of $(D, \gamma)$ and $\phi$.

Fix a path $\tilde{\gamma}$, a nondegenerate disc $(D, \gamma)$, and a map $\phi: D \rightarrow X / G$ as above. Since $X / G$ is $2-$ connected, Theorem 2.9 and the fact that any two triangulations of a closed 2-disc have a common subdivision imply that it is enough to show that the output of the Armstrong construction is invariant under elementary subdivisions and elementary pushes. The arguments for these are similar; we will give the details for elementary pushes and leave the case of elementary subdivisions to the reader.

Let $y$ be a vertex of $D$ that does not lie on $\gamma$. Set $z=\phi(y)$ and let $z^{\prime} \in X / G$ be any vertex such that for all simplices $s$ of $\operatorname{Star}_{D}(y)$, the set $\phi(s) \cup z^{\prime}$ is a simplex of $X / G$. Define $\phi^{\prime}: D \rightarrow X / G$ to equal $\phi$ on all vertices except for $y$, where it equals $z^{\prime}$. Our goal is to show that there exist full sequences of moves

$$
\phi:(D, \gamma, \tilde{\gamma}) \rightarrow \cdots
$$

and

$$
\phi^{\prime}:(D, \gamma, \tilde{\gamma}) \rightarrow \cdots
$$

such that the stabilizer products of (20) and (21) are equal.

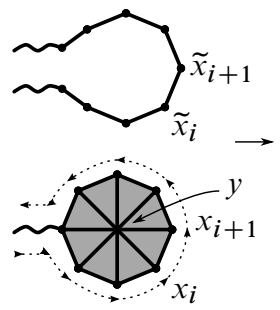

(a)
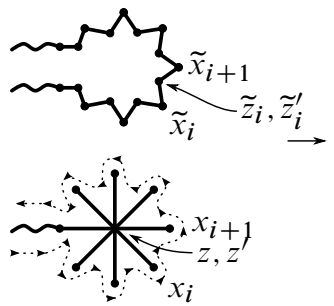

(b)
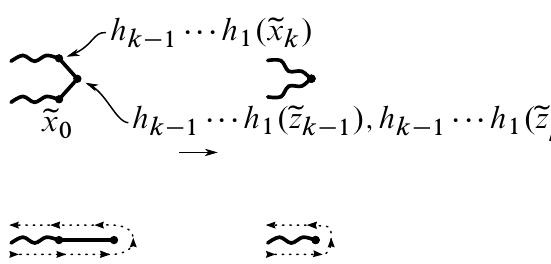

(c) (d)

Figure 7: (a) Bottom is $(D, \gamma)$, top is $\tilde{\gamma}$ (b) Bottom is $(E, \delta)$, top is $\tilde{\delta}$ and $\tilde{\delta}^{\prime} \quad$ (c) Bottom is $(F, \epsilon)$, top is $\tilde{\epsilon}$ and $\tilde{\epsilon}^{\prime}$ (d) Bottom is $(C, \eta)$, top is $\tilde{\eta}$

By Corollary 2.7, we can assume that $(D, \gamma)$ is a flower whose bloom is $\operatorname{Star}_{D}(y)$ (see Figure 7(a)). Let the images under $\phi$ and $\phi^{\prime}$ of the portion of $\gamma$ that goes around the bloom (these are equal) be

$$
x_{0}-x_{1}-\cdots-x_{k}=x_{0},
$$

and let the corresponding subpath of $\tilde{\gamma}$ be

$$
\tilde{x}_{0}-\tilde{x}_{1}-\cdots-\tilde{x}_{k}
$$


For $0 \leq i<k$, let $\widetilde{z}_{i}$ be a lift of $z$ to $X$ and $\widetilde{z}_{i}^{\prime}$ be a lift of $z^{\prime}$ to $X$ such that $\left\{\tilde{x}_{i}, \tilde{x}_{i+1}, \tilde{z}_{i}, \tilde{z}_{i}^{\prime}\right\}$ is a simplex of $X$. Define $(E, \delta)$ to be as depicted in Figure 7(b). Let $\tilde{\delta}$ and $\tilde{\delta}^{\prime}$ to be $\tilde{\gamma}$ with the subpath (22) replaced with the paths

$$
\begin{aligned}
& \tilde{x}_{0}-\tilde{z}_{0}-\tilde{x}_{1}-\tilde{z}_{1}-\tilde{x}_{2}-\cdots-\tilde{z}_{k-1}-\tilde{x}_{k} \\
& \text { and } \quad \tilde{x}_{0}-\widetilde{z}_{0}^{\prime}-\tilde{x}_{1}-\widetilde{z}_{1}^{\prime}-\tilde{x}_{2}-\cdots-\widetilde{z}_{k-1}^{\prime}-\tilde{x}_{k},
\end{aligned}
$$

respectively (see Figure 7(b)). Observe that there is a sequence of lifted 2-dimensional collapses which converts $(D, \gamma, \tilde{\gamma})$ to $(E, \delta, \tilde{\delta})$, and similarly there is a sequence of lifted 2-dimensional collapses which converts $(D, \gamma, \tilde{\gamma})$ to $\left(E, \delta, \widetilde{\delta^{\prime}}\right)$.

Now let $(F, \epsilon)$ equal the degenerate disc depicted in Figure $7(\mathrm{c})$; it is obtained by performing one-dimensional collapses to the "spokes" of $E$. We lift these one-dimensional collapses as follows. First, we choose $h_{1} \in G_{\widetilde{x}_{1}}$ such that $h_{1}\left(\widetilde{z}_{1}\right)=\widetilde{z}_{0}$ and $h_{1}\left(\widetilde{z}_{1}^{\prime}\right)=\widetilde{z}_{0}^{\prime}$; such a choice is possible since $\left\{\widetilde{x}_{1}, \widetilde{z}_{0}, \widetilde{z}_{0}^{\prime}\right\}$ and $\left\{\widetilde{x}_{1}, \widetilde{z}_{1}, \widetilde{z}_{1}^{\prime}\right\}$ are both simplices of $X$. We then swing $\tilde{\delta}$ and $\tilde{\delta}^{\prime}$ around the pivot point $\tilde{x}_{1}$ by $h_{1}$ and delete $\tilde{x}_{1}$. Next, we choose $h_{2} \in G_{h_{1}\left(\tilde{x}_{1}\right)}$ such that $h_{2}\left(h_{1}\left(\widetilde{z}_{2}\right)\right)=\widetilde{z}_{0}$ and $h_{2}\left(h_{1}\left(\widetilde{z}_{2}^{\prime}\right)\right)=\widetilde{z}_{0}^{\prime}$; such a choice is possible since both

$$
\begin{aligned}
h_{1}\left(\left\{\tilde{x}_{2}, \tilde{z}_{2}, \widetilde{z}_{2}^{\prime}\right\}\right) & =\left\{h_{1}\left(\tilde{x}_{2}\right), h_{1}\left(\widetilde{z}_{2}\right), h_{1}\left(\widetilde{z}_{2}^{\prime}\right)\right\} \\
\text { and } \quad & h_{1}\left(\left\{\tilde{x}_{2}, \tilde{z}_{1}, \widetilde{z}_{1}^{\prime}\right\}\right)=\left\{h_{1}\left(\tilde{x}_{2}\right), \widetilde{z}_{0}, \widetilde{z}_{0}^{\prime}\right\}
\end{aligned}
$$

are simplices of $X / G$. We then swing $\tilde{\delta}$ and $\tilde{\delta}^{\prime}$ around the pivot point $h_{1}\left(\tilde{x}_{2}\right)$ by $h_{2}$ and delete $h_{1}\left(\tilde{x}_{2}\right)$. Continuing in this manner, we obtain lifts $\tilde{\epsilon}$ and $\tilde{\epsilon}^{\prime}$ of $\epsilon$ such that there are sequences of one-dimensional collapses

$$
\begin{aligned}
& \left.\phi\right|_{E}:(E, \delta, \tilde{\delta}) \rightarrow \cdots \rightarrow(F, \epsilon, \tilde{\epsilon}) \\
& \text { and }\left.\quad \phi^{\prime}\right|_{E}:\left(E, \delta, \tilde{\delta}^{\prime}\right) \rightarrow \cdots \rightarrow\left(F, \epsilon, \tilde{\epsilon}^{\prime}\right)
\end{aligned}
$$

satisfying the following two conditions.

- In (23) and (24), the paths are swung around the same pivot points by the same group elements.

- The paths $\tilde{\epsilon}$ and $\tilde{\epsilon}^{\prime}$ are as depicted in Figure 7(c).

Observe that the sets

$$
\begin{array}{ll} 
& \left\{h_{k-1} \cdots h_{1}\left(\widetilde{x}_{k}\right), h_{k-1} \cdots h_{1}\left(\widetilde{z}_{k-1}\right), h_{k-1} \cdots h_{1}\left(\widetilde{z}_{k-1}^{\prime}\right)\right\} \\
\text { and } \quad & \left\{\widetilde{x}_{0}, h_{k-1} \cdots h_{1}\left(\widetilde{z}_{k-1}\right), h_{k-1} \cdots h_{1}\left(\widetilde{z}_{k-1}^{\prime}\right)\right\}
\end{array}
$$

are simplices of $X / G$. Thus there exists some

$$
h_{k} \in G_{\left\{h_{k-1} \cdots h_{1}\left(\widetilde{z}_{k-1}\right), h_{k-1} \cdots h_{1}\left(\widetilde{z}_{k-1}^{\prime}\right)\right\}}
$$


such that $h_{k} h_{k-1} \cdots h_{1}\left(\tilde{x}_{k}\right)=\tilde{x}_{0}$. Let $(C, \eta)$ be the degenerate disc depicted in Figure $7(\mathrm{~d})$ and let $\tilde{\eta}$ be the result of swinging $\tilde{\epsilon}$ around $h_{k-1} \cdots h_{1}\left(\widetilde{z}_{k-1}\right)$ by $h_{k}$ and then deleting $h_{k-1} \cdots h_{1}\left(\widetilde{z}_{k-1}\right)$ (see Figure $7(\mathrm{~d})$ ). Observe that $\tilde{\eta}$ also equals the result of swinging $\widetilde{\epsilon}^{\prime}$ around $h_{k-1} \cdots h_{1}\left(\widetilde{z}_{k-1}^{\prime}\right)$ by $h_{k}$ and then deleting $h_{k-1} \cdots h_{1}\left(\widetilde{z}_{k-1}^{\prime}\right)$.

Summing up, we have constructed sequences of moves

$$
\begin{aligned}
& \phi:(D, \gamma, \tilde{\gamma}) \rightarrow \cdots \rightarrow(E, \delta, \tilde{\delta}) \rightarrow \cdots \rightarrow(F, \epsilon, \widetilde{\epsilon}) \stackrel{h_{k}, h_{k-1} \cdots h_{1}\left(\tilde{z}_{k-1}\right)}{\longrightarrow}(C, \eta, \tilde{\eta}) \\
& \phi^{\prime}:(D, \gamma, \tilde{\gamma}) \rightarrow \cdots \rightarrow\left(E, \delta, \tilde{\delta}^{\prime}\right) \rightarrow \cdots \rightarrow\left(F, \epsilon, \tilde{\epsilon}^{\prime}\right) \stackrel{h_{k}, h_{k-1} \cdots h_{1}\left(\widetilde{z}_{k-1}^{\prime}\right)}{\longrightarrow}(C, \eta, \widetilde{\eta})
\end{aligned}
$$

Moreover, in both of these sequences the pivot points and group elements are all identical except for the final ones. Since $\widetilde{z}_{k-1}$ and $\widetilde{z}_{k-1}^{\prime}$ are joined by an edge, we have an edge relation

$$
\left(h_{k}\right)_{h_{k-1} \cdots h_{1}\left(\widetilde{z}_{k-1}\right)}=\left(h_{k}\right)_{h_{k-1} \cdots h_{1}\left(\widetilde{z}_{k-1}^{\prime}\right)}
$$

in $\Gamma$. Thus the stabilizer products of (25) and (26) are equal, and we are done.

\subsubsection{The output of the Armstrong construction does not depend on choice 1 (the} path $\tilde{\gamma}$ from $\tilde{v}$ to $g(\widetilde{v})$ ) By the previous two sections, the output of the Armstrong construction only depends on the path $\tilde{\gamma}$ from $\tilde{v}$ to $g(\widetilde{v})$. We wish to show that in fact it is independent of this path.

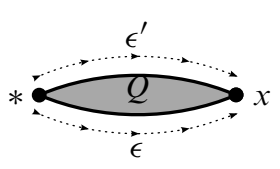

(a)

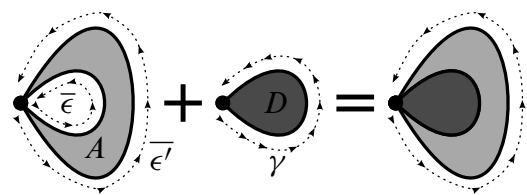

(b)

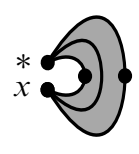

(c)

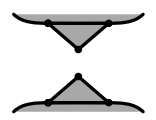

(d)

Figure 8: (a) $(Q, *)$ (b) Gluing $A$ and $D$ to get $D^{\prime} \quad$ (c) Gluing $*$ to $x$ does not result in a simplicial complex (d) Gluing top to bottom does not result in a simplicial complex

Consider any two simplicial paths $\tilde{\gamma}$ and $\tilde{\gamma}^{\prime}$ from $\tilde{v}$ to $g(\widetilde{v})$. We will construct nondegenerate discs $(D, \gamma)$ and $\left(D^{\prime}, \gamma^{\prime}\right)$ together with simplicial maps $\phi: D \rightarrow X / G$ and $\phi^{\prime}: D^{\prime} \rightarrow X / G$ such that $\phi_{*}(\gamma)=\pi_{*}(\tilde{\gamma})$ and $\phi_{*}^{\prime}\left(\gamma^{\prime}\right)=\pi_{*}\left(\tilde{\gamma}^{\prime}\right)$. Additionally, we will construct full sequences of moves

$$
\begin{array}{ll} 
& \phi:(D, \gamma, \tilde{\gamma}) \rightarrow \cdots \\
\text { and } \quad & \phi:\left(D^{\prime}, \gamma^{\prime}, \tilde{\gamma}^{\prime}\right) \rightarrow \cdots
\end{array}
$$

such that the stabilizer products of (27) and (28) are equal. This will imply the desired result. 
Since $X$ is simply connected, $\tilde{\gamma}$ and $\tilde{\gamma}^{\prime}$ are homotopic fixing the endpoints. This implies that there exists a based simplicial complex $(Q, *)$ such that $Q$ is homeomorphic to a closed 2-disc and $* \in \partial Q$ together with a map $\tilde{\rho}: Q \rightarrow X$ with the following property. There is a vertex $x$ on $\partial Q$ such that if $\epsilon$ and $\epsilon^{\prime}$ are the two embedded paths in $\partial Q$ from $*$ to $x$, then $\tilde{\rho}_{*}(\epsilon)=\tilde{\gamma}$ and $\tilde{\rho}_{*}\left(\epsilon^{\prime}\right)=\tilde{\gamma}^{\prime}$ (see Figure 8(a)). Subdividing $Q$ if necessary away from $\partial Q$, we can glue $x$ and $*$ together to get a simplicial complex $A$ (see Figure 8(c) for an example of why we may need to subdivide $Q$ first). Observing that $\pi(\widetilde{\rho}(x))=\pi(\widetilde{\rho}(*))=v$, let $\rho: A \rightarrow X / G$ be the map induced by by $\pi \circ \tilde{\rho}: Q \rightarrow X / G$ (see Figure 8(b)) and let the projections of the paths $\epsilon$ and $\epsilon^{\prime}$ in $Q$ to $A$ be $\bar{\epsilon}$ and $\bar{\epsilon}^{\prime}$, respectively.

Choose a nondegenerate disc $(D, \gamma)$ and a map $\phi: D \rightarrow X / G$ such that $\phi_{*}(\gamma)=\pi_{*}(\tilde{\gamma})$. Subdividing $D$ away from $\gamma$ and $A$ away from $\bar{\epsilon}$ and $\bar{\epsilon}^{\prime}$ if necessary, glue $\bar{\epsilon} \subset A$ to $\gamma \subset D$ to obtain a simplicial complex $D^{\prime}$ which is homeomorphic to a closed two-disc (see Figure 8(b); also see Figure 8(d) for an example of why we may need to subdivide). Let $\gamma^{\prime}$ be the loop around the boundary of $D^{\prime}$ corresponding to $\bar{\epsilon}^{\prime}$. Thus $\left(D^{\prime}, \gamma^{\prime}\right)$ is another nondegenerate disc. The maps $\phi$ and $\rho$ induce a map $\phi^{\prime}: D^{\prime} \rightarrow X / G$ such that $\phi_{*}^{\prime}\left(\gamma^{\prime}\right)=\pi_{*}\left(\tilde{\gamma}^{\prime}\right)$. Now, by Corollary 2.7, there is a sequence of collapses which converts $\left(D^{\prime}, \gamma^{\prime}\right)$ into $(D, \gamma)$. Moreover, by construction there is a partial sequence of moves

$$
\phi^{\prime}:\left(D^{\prime}, \gamma^{\prime}, \tilde{\gamma}^{\prime}\right) \rightarrow \cdots \rightarrow(D, \gamma, \tilde{\gamma})
$$

in which no swinging occurs (and, in particular, whose stabilizer product is trivial). Letting

$$
\phi:(D, \gamma, \tilde{\gamma}) \rightarrow \cdots
$$

be any full sequence of moves across $(D, \gamma, \tilde{\gamma})$, we can append (29) to the beginning of (30) to obtain a full sequence of moves

$$
\phi^{\prime}:\left(D^{\prime}, \gamma^{\prime}, \tilde{\gamma}^{\prime}\right) \rightarrow \cdots \rightarrow(D, \gamma, \tilde{\gamma}) \rightarrow \cdots .
$$

By construction, the stabilizer products of (30) and (31) are equal, and we are done.

\subsection{Proof that the Armstrong construction defines a surjective homomor- phism}

In Section 3.2, we showed that the Armstrong construction gives a well-defined map $\psi^{-1}: G \rightarrow \Gamma$. It is clear that $\psi^{-1}$ is a homomorphism. We must check that it surjective and that $\psi \circ \psi^{-1}=1$. Observe that $\Gamma$ is generated by the set

$$
S=\left\{g_{\tilde{x}} \mid \tilde{x} \in X^{(0)} \text { and } g \in G_{\tilde{x}}\right\} .
$$


Consider $g_{\tilde{x}} \in S$. It is enough to show that $\psi^{-1}(g)=g_{\tilde{x}}$.

Let $\tilde{\gamma}^{\prime}$ be a path in $X$ from $\tilde{v}$ to $\tilde{x}$ and let $\left(\tilde{\gamma}^{\prime}\right)^{-1}$ be $\tilde{\gamma}^{\prime}$ traversed in reverse order. We then obtain a path $\tilde{\gamma}$ in $X$ from $\tilde{v}$ to $g(\widetilde{v})$ by concatenating $\tilde{\gamma}^{\prime}$ with $g\left(\left(\tilde{\gamma}^{\prime}\right)^{-1}\right)$. Observe that $\pi_{*}(\tilde{\gamma})$ is the image of the boundary of a line. We can construct a full sequence of moves across this line by first swinging $\tilde{\gamma}$ around $\tilde{x}$ by $g^{-1}$ and then doing a sequence of one-dimensional collapses without any additional swinging. This implies that $\psi^{-1}(g)=g_{\tilde{x}}$, and we are done.

\section{References}

[1] M A Armstrong, On the fundamental group of an orbit space, Proc. Cambridge Philos. Soc. 61 (1965) 639-646 MR0187244

[2] M A Armstrong, A presentation for a group of automorphisms of a simplicial complex, Glasgow Math. J. 30 (1988) 331-337 MR964580

[3] B van den Berg, On the Abelianization of the Torelli group, doctoral thesis, University of Utrecht (2003)

[4] R H Bing, Some aspects of the topology of 3-manifolds related to the Poincaré conjecture, from: "Lectures on modern mathematics, Vol II", Wiley, New York (1964) 93-128 MR0172254

[5] A Björner, M Wachs, On lexicographically shellable posets, Trans. Amer. Math. Soc. 277 (1983) 323-341 MR690055

[6] M R Bridson, A Haefliger, Metric spaces of non-positive curvature, Grundlehren der Mathematischen Wissenschaften 319, Springer, Berlin (1999) MR1744486

[7] K S Brown, Presentations for groups acting on simply-connected complexes, J. Pure Appl. Algebra 32 (1984) 1-10 MR739633

[8] K S Brown, Cohomology of groups, Graduate Texts in Mathematics 87, Springer, New York (1994) MR1324339 Corrected reprint of the 1982 original

[9] R E Goodrick, Non-simplicially collapsible triangulations of $I^{n}$, Proc. Cambridge Philos. Soc. 64 (1968) 31-36 MR0220272

[10] A Hatcher, W Thurston, A presentation for the mapping class group of a closed orientable surface, Topology 19 (1980) 221-237 MR579573

[11] D Johnson, The structure of the Torelli group I: A finite set of generators for I, Ann. of Math. (2) 118 (1983) 423-442 MR727699

[12] D Johnson, The structure of the Torelli group III: The abelianization of $\mathcal{T}$, Topology 24 (1985) 127-144 MR793179

[13] H Maazen, Homology stability for the general linear group, doctoral thesis, University of Utrecht (1979) 
[14] J McCool, Some finitely presented subgroups of the automorphism group of a free group, J. Algebra 35 (1975) 205-213 MR0396764

[15] D McCullough, A Miller, The genus 2 Torelli group is not finitely generated, Topology Appl. 22 (1986) 43-49 MR831180

[16] G Mess, The Torelli groups for genus 2 and 3 surfaces, Topology 31 (1992) 775-790 MR1191379

[17] J Milnor, Introduction to algebraic $K$-theory, Annals of Mathematics Studies 72, Princeton University Press, Princeton, N.J. (1971) MR0349811

[18] A Putman, Presentations for the level 2 and 3 congruence subgroups of $\mathrm{SL}_{n}(\mathbb{Z})$, in preparation

[19] A Putman, An infinite presentation of the Torelli group, Geom. Funct. Anal. 19 (2009) 591-643 MR2545251

[20] J-P Serre, Trees, Springer, Berlin (1980) MR607504 Translated from the French by John Stillwell

[21] EH Spanier, Algebraic topology, McGraw-Hill Book Co., New York (1966) MR0210112

Department of Mathematics, Rice University

6100 Main St., Houston TX 77005, USA

andyp@rice.edu

http://www.math.rice.edu/ andyp/

Received: 6 January 2011 